\title{
Overexpression of Mstl reduces gastric cancer cell viability by repressing the AMPK-Sirt3 pathway and activating mitochondrial fission
}

This article was published in the following Dove Press journal: OncoTargets and Therapy

\author{
Shiwei Yaol,* \\ Wei Yan ${ }^{2, *}$ \\ 'Department of Gastroenterology, \\ Beijing Tiantan Hospital Affiliated \\ to Capital Medical University, \\ Beijing, China; ${ }^{2}$ Department of \\ Gastroenterology, The First Hospital \\ of Tsinghua University, Beijing, China \\ *These authors contributed equally \\ to this work
}

Background and objective: Mammalian sterile 20-like kinase 1 (Mst1) plays a critical role in regulating cell survival and apoptosis. However, its influence on gastric cancer cell viability is not understood. Our study aims to explore the specific role of Mst1 in gastric cancer.

Materials and methods: Cellular viability was measured via TUNEL staining, MTT assays, and Western blotting. Immunofluorescence was performed to observe mitochondrial fission. Mst1 overexpression assays were conducted to observe the regulatory mechanisms of Mst1 in mitochondrial fission and cell apoptosis.

Results: The results demonstrated that Mst1 was downregulated in AGS cells when compared with GES-1 cells. However, overexpression of Mst1 reduced cell viability and increased apoptosis in AGS cells. Molecular experiments showed that Mst1 overexpression mediated mitochondrial damage, as evidenced by decreased ATP production, increased ROS generation, more cyt-c translocation from the mitochondria into the cytoplasm and nucleus, and activated the caspase-9-related apoptotic pathway. Furthermore, we found that mitochondrial fission was required for Mst1-induced mitochondrial dysfunction; inhibition of mitochondrial fission sustained mitochondrial homeostasis in response to Mst1 overexpression. In addition, our data revealed that Mst1 controlled mitochondrial fission via repressing the AMPK-Sirt3 pathway. Activation of the AMPK-Sirt3 pathway negated the promoting effect of Mst1 overexpression on mitochondrial fission.

Conclusion: Altogether, our data identified Mst1 as a novel tumor-suppressive factor in promoting cell death in gastric cancer cells by triggering mitochondrial fission and blocking the AMPK-Sirt3 axis.

Keywords: Mst1, gastric cancer, mitochondrial fission, apoptosis, AMPK-Sirt3 pathway

\section{Introduction}

Gastric cancer, the most common cancer throughout the world, is the second leading cause of cancer-related mortality worldwide. ${ }^{1}$ The risk factors for stomach cancer include older age, Helicobacter pylori infection, smoking, gastroesophageal reflux disease, low consumption of fresh fruits and vegetables, and high intake of salty and smoked foods. ${ }^{2}$ Although there is a rapid advance in the diagnosis and treatment of gastric cancer, the molecular pathogenesis of gastric cancer has not been adequately investigated.

Mammalian sterile 20-like kinase 1 (Mst1) is a kind of mitogen-activated protein kinase-related kinase that plays a key role in regulating cell apoptosis and survival. ${ }^{3,4}$ In response to the activation of the caspase family, Mst1 is stimulated and contributes to the phosphorylation of histone, which promotes the DNA breakage that evokes
Correspondence: Shiwei Yao

Department of Gastroenterology,

Beijing Tiantan Hospital Affiliated

to Capital Medical University,

No I19, South Fourth Ring West Road,

Fengtai District, Beijing 100050, China

Tel +861067096644

Email wei_newl@sina.com
OncoTargets and Therapy 20 I 8: I / 8465-8479

Dovepress if in 8

http://dx,doi.org/10.214710TTS180851 (c) (1) (-) 2018 Yao and Yan. This work is published and licensed by Dove Medical Press Limited. The full terms of this license are available at https://www.dovepress.com/terms.php cc) and incorporate the Creative Commons Attribution - Non Commercial (unported, v3.0) License (http://creativecommons.org/licenses/by-nc/3.0/). By accessing the work you hereby accept the Terms. Non-commercial uses of the work are permitted without any further permission from Dove Medical Press Limited, provided the work is properly attributed. For permission for commercial use of this work, please see paragraphs 4.2 and 5 of our Terms (https://www.dovepress.com/terms.php). 
cellular death via apoptosis. ${ }^{5}$ Ample evidence is available to establish the crucial role played by Mst1 in regulating tissue size and limiting cancer development. For example, higher expression of Mst 1 is associated with a decrease in the proliferation of pancreatic cancer cells. ${ }^{6}$ Moreover, Mst1 has been identified as a potential early detection biomarker for the development of colorectal cancer. ${ }^{7}$ Increased Mst1 promotes glioma via modifying the TGF $\beta$ pathway. ${ }^{8}$ Overexpression of Mst1 augments liver cancer death through regulating the Wnt/ $\beta$-catenin pathway. ${ }^{9}$ Notably, the expression of Mst1 is significantly downregulated in patients with gastric cancer. ${ }^{10}$ However, no study has examined the functional influence of Mst1 in gastric cancer cell viability.

It is currently clear that mitochondria are significant for oncogenesis. Mitochondria participate in tumor bioenergetics, control the cellular redox balance, regulate cancer invasion by modulating cellular calcium homeostasis, and mediate cell death via apoptosis or necrosis. ${ }^{1-13}$ Accumulating evidence confirms the necessary role of mitochondria in tumor development, progress and response to therapy. Interest in the role of mitochondrial fission in cancer originated with the demonstration that mitochondrial fission plays a decisive step in regulating mitochondrial integrity and cancer viability via multiple effects. ${ }^{14}$ For example, mitochondrial fission induces excessive production of ROS, shaping cellular oxidative stress. ${ }^{15,16}$ Moreover, mitochondrial ATP production is also handled by mitochondrial fission. ${ }^{17}$ Uncontrolled mitochondrial fission directly activates the caspase-9-related cell apoptotic pathway. Considering the link between Mst1 and mitochondrial apoptosis, we question whether Mst1 modulates gastric cancer death via coping with mitochondrial fission.

Previous studies have reported that mitochondrial fission is primarily regulated by two pathways: the AMPK axis ${ }^{18}$ and the MAPK-JNK cascade. ${ }^{19}$ In diabetic cardiomyopathy and energy shortage, AMPK is inactivated and promotes mitochondrial fission, modifying bioenergetic metabolism and cell death. ${ }^{20}$ Acute stress can initiate mitochondrial fission via the JNK pathway with activating phosphorylation of mitochondrial fission-related factors. ${ }^{21}$ In the present study, we explore the role of the AMPK axis in Mst1mediated mitochondrial fission in gastric cancer. As the downstream effector of AMPK, Sirt3 has been found to exert an inhibitory effect on cancer growth and metastasis by controlling mitochondrial homeostasis. ${ }^{22}$ Elevated Sirt3 blocks mitochondrial fission in cerebral ischemia-reperfusion via suppressing the $\mathrm{Wnt} / \beta$-catenin pathway. ${ }^{23}$ In contrast, Sirt3 deficiency exacerbates p53-related mitochondrial dysfunction in aged hearts. ${ }^{24}$ However, whether Mst1 has a role in regulating mitochondrial fission via the AMPK-Sirt3 pathway is not clear. Collectively, the aim of our study is to explore the action and mechanism of Mst1 in gastric cancer cell viability, with a focus on mitochondrial fission and its regulatory signal, the AMPK-Sirt3 pathway.

\section{Materials and methods Cell lines and culture}

The AGS gastric cancer cell line (AGS cells, ATCC ${ }^{\circledR}$ CRL-1739'TM) was purchased from the American Type Culture Collection. The GES-1 normal gastric mucosal cell line (GES-1 cells) was obtained from the Cell Bank of the Chinese Academy of Sciences. These cells were cultured in L-DMEM supplemented with $10 \% \mathrm{FBS}$ and $1 \%$ penicillin/streptomycin at $37^{\circ} \mathrm{C}$ in a humidified atmosphere with $5 \%$ of $\mathrm{CO}_{2}$. To perform the loss- and gain-of-function assays for mitochondrial fission, Mdivi-1 (10 mM; Merck KGaA, Darmstadt, Germany) and FCCP (5 $\mu \mathrm{m}$, Selleck Chemicals, Houston, TX, USA) were pre-incubated with cells for 2 hours. To activate and inhibit the AMPK pathway, AICAR (AI; Merck KGaA, Cat Number 2627-69-2) and Compound C (Selleck Chemicals) were administered into the cell medium for 2 hours.

\section{MTT assay for cellular viability and mitochondrial permeability transition pore (mPTP) opening rate detection}

The cell viability was determined by MTT assays (Merck $\mathrm{KGaA}$ ). Briefly, cells were seeded onto 96-well plates, and then $20 \mu \mathrm{L}$ of MTT at a concentration of $5 \mathrm{mg} / \mathrm{mL}$ was added to the medium. The plates were placed for 4 hours in the dark at $37^{\circ} \mathrm{C}$ and $5 \% \mathrm{CO}_{2}$. After that, the medium was removed and $100 \mu \mathrm{L}$ dimethyl sulfoxide (DMSO) was added into the medium for 15 minutes in the dark at $37^{\circ} \mathrm{C}$ and $5 \%$ $\mathrm{CO}_{2}$. Then, the samples were observed at a wavelength of $570 \mathrm{~nm}$. The relative cell viability was recorded as a ratio to that of the control group. ${ }^{25}$

To measure the MPTP opening, cells were loaded with PBS containing $25 \mathrm{nM}$ tetramethylrhodamine methyl ester (TMRM; Thermo Fisher Scientific, Waltham, MA, USA, T668). After 30 minutes, cells were washed with PBS again to remove the free TMRM. Then, samples were observed at a wavelength of $480 \mathrm{~nm}$ using a microplate reader (Epoch 2; BioTek Instruments, Inc., Winooski, VT, USA). Cells treated with PBS were used as the control group for MTT assay and TUNEL staining. ${ }^{26}$

\section{Cellular ATP quantification and mitochondrial membrane potential staining} Cellular ATP was determined using the Enhanced ATP Assay Kit (Beyotime, Shanghai, China, Cat. No: S0027). 
Briefly, cells were lysed using RIPA Lysis Buffer (Beyotime, Cat. No: P0013E), and then, the protein concentration was assessed using the Enhanced BCA Protein Assay Kit (Beyotime, Cat. No: P0009). ATP was detected according to the manufacturer's protocol. ${ }^{27}$ Mitochondrial membrane potential was stained using the Mitochondrial Membrane

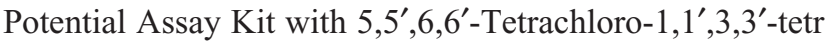
aethylbenzimidazolylcarbocyanine iodide (JC-1) (Beyotime, Cat. No: C2006). Cells were seeded in a 24-well plate at a density of $1 \times 10^{4}$ cells per well. Then, $10 \mu \mathrm{L}$ of JC- 1 solution was added into the medium for 30 minutes under $5 \%$ $\mathrm{CO}_{2}$ at $37^{\circ} \mathrm{C}$. After that, PBS was used to wash the cells to remove the free JC-1 probe. The mitochondrial membrane potential was observed under a laser confocal microscope (TcS SP5; Leica Microsystems, Inc., Buffalo Grove, IL, USA). The relative fluorescence intensity was estimated. The alteration of mitochondrial membrane potential was presented as a ratio of red-to-green fluorescence.

\section{Cell death detection and EdU assay}

Cell death was determined via the lactate dehydrogenase (LDH) release assay, terminal deoxynucleotidyl transferase dUTP nick end labeling (TUNEL) staining, and caspase protein activity assay. The TUNEL experiment was carried out to stain apoptotic cells. Briefly, cells were fixed with $4 \%$ paraformaldehyde in PBS at room temperature for 30 minutes and then permeabilized with $0.2 \%$ Triton X-100 for $30 \mathrm{~min}$ utes at room temperature. Subsequently, the samples were incubated with TUNEL solution at $37^{\circ} \mathrm{C}$ for $1 \mathrm{~h}$. After being washed with PBS, the samples were counterstained with 4',6-diamidino-2-phenylindole (DAPI). The laser confocal microscope (TcS SP5; Leica Microsystems, Inc.) was used to observe the apoptotic cells, and the percentage of TUNELpositive cells was measured by counting at least 200 cells from random fields of view. LDH release was determined using the LDH Cytotoxicity Assay Kit (Beyotime, Cat. No: C0016) according to the manufacturer's protocol. ${ }^{28}$ Caspase- 3 and caspase-9 activities were analyzed using the following commercial kits: Caspase-3 Activity Assay Kit (Beyotime, Cat. No: C1115) and Caspase-9 Activity Assay Kit (Beyotime, Cat. No: C1158) according to the manufacturer's instructions.

EdU staining was conducted using the BeyoClick ${ }^{\mathrm{TM}}$ EdU Cell Proliferation Kit with Alexa Fluor 594 (Beyotime, Cat. No: C00788L). Cells were washed with PBS. Fresh DMEM was added, and then, $10 \mu \mathrm{M}$ EdU was added into the medium. The cells were incubated for 2 hours at $37^{\circ} \mathrm{C} / 5 \%$ $\mathrm{CO}_{2}$. After the incubation, the cells were washed with PBS to remove the DMEM and the free EdU probe. The cells were then fixed in $4 \%$ paraformaldehyde at room temperature for
30 minutes before being stained with DAPI for 3 minutes. After an additional wash in PBS, the cells were observed under an inverted microscope. ${ }^{29}$

\section{Western blotting}

Total proteins were extracted using RIPA Lysis Buffer (Beyotime, Cat. No: P0013E). After that, proteins were rapidly centrifuged $(20,000 \mathrm{rpm})$ for $10 \mathrm{~min}$ at $4^{\circ} \mathrm{C}$ to pellet cell debris. Supernatant was collected and quantified using an Enhanced BCA Protein Assay Kit (Beyotime, Cat. No: P0009). Then, proteins (45-60 $\mu \mathrm{g}$ ) were loaded in a 10\%-15\% SDS-PAGE gel and transferred to polyvinylidene difluoride (PVDF) membranes (Bio-Rad Laboratories, Inc., Hercules, CA, USA). Subsequently, membranes were blocked with 5\% skim milk for 45 minutes at room temperature. After washing with tris-buffered saline and polysorbate 20 (TBST) three times at room temperature, the membranes were incubated with the primary antibodies at $4{ }^{\circ} \mathrm{C}$ overnight. The primary antibodies used in the present study are described below: caspase-9 (1:1,000, Cell Signaling Technology, Inc., Danvers, MA, USA, \#9504), pro-caspase-3 (1:1,000, Abcam, Cambridge, UK, \#ab13847), cleaved caspase-3 (1:1,000, Abcam, \#ab49822), c-IAP (1:1,000, Cell Signaling Technology, Inc., \#4952), Bad (1:1,000, Abcam, \#ab90435), cyt-c (1:1,000, Abcam, \#ab90529), Drp1 (1:1,000, Abcam, \#ab56788), Fis 1 (1:1,000, Abcam, \#ab71498), Parkin (1:1,000, Cell Signaling Technology, Inc.), Opa1 (1:1,000, Abcam, \#ab42364), Mfn2 (1:1,000, Abcam, \#ab56889), Mff (1:1,000, Cell Signaling Technology, Inc., \#86668), AMPK (1:1,000, Abcam, \#ab131512), p-AMPK (1:1,000, Abcam, \#ab23875), Mst1 (1:1,000, Cell Signaling Technology, Inc., \#3682), Sirt3 (1:1,000, Abcam, \#ab86671), complex III subunit core (CIII-core2, 1:1,000, Invitrogen, Carlsbad, CA, USA, \#459220), complex II (CII-30, 1:1,000, Abcam, \#ab110410), complex IV subunit II (CIV-II, 1:1,000, Abcam, \#ab1 10268). After being washed with TBST three times, the membranes were further incubated with the anti-mouse or anti-rabbit secondary antibody for 45 minutes at room temperature. The proteins were visualized using Pierce enhanced chemiluminescence Western blotting substrate (Pierce; Thermo Fisher Scientific). ${ }^{30}$ Mean densities of the bands were evaluated and normalized to that of $\beta$-actin (Quantity One, version 4.6.2; Bio-Rad Laboratories, Inc.).

\section{Measurement of cellular redox balance}

Cellular reactive oxygen species (ROS) generation was quantified using flow cytometry. Cells were seeded onto the 12-well plates. After washing with PBS, dihydroethidium (DHE) staining was added into the medium and the cells 
were incubated with the DHE probe for 30 minutes in the dark at $37^{\circ} \mathrm{C}$ and $5 \% \mathrm{CO}_{2}$. Then, PBS was used to wash cells to remove the free DHE probe. Subsequently, $0.25 \%$ trypsin was applied to collect the cell. Flow cytometry analysis was performed using the FACSCanto II cytometer (BD FACSCanto II; BD Biosciences, San Jose, CA, USA). Analysis of the data was performed using FACSDiva software (BD Biosciences). The ROS production was also observed using a laser confocal microscope (TcS SP5; Leica Microsystems, Inc.). The concentration of cellular antioxidant factors such as glutathione (GSH; Glutathione Reductase Assay Kit; Beyotime, Cat. No S0055), superoxide dismutase (SOD; Total Superoxide Dismutase Assay Kit; Beyotime, Cat. No S0101), and glutathione peroxidase (GPX; Cellular Glutathione Peroxidase Assay Kit; Beyotime, Cat. No S0056) were measured via ELISA according to the manufacturer's guidelines. ${ }^{31}$

\section{Immunofluorescence and mitochondrial fission detection}

Cells were seeded onto poly-D-lysine coated coverslips. ${ }^{32}$ Then, methanol-free 4\% paraformaldehyde was used to fix cells for 15 minutes at room temperature. Subsequently, samples were blocked with 5\% goat serum at room temperature for 45 minutes. After washing with TBST, samples were incubated with primary antibody at $4^{\circ} \mathrm{C}$ overnight. The primary antibodies used in the present study were as follows: p-AMPK (1:1,000, Abcam, \#ab23875), Sirt3 (1:1,000, Abcam, no ab86671) and Mst1 (1:1,000, Cell Signaling Technology, Inc., \#3682). Mitochondrial fission was quantified via measuring the length of mitochondria according to the previous studies. At least 200 cells with tubular, fragmented, intermediate mitochondria were observed, and then, the average length of mitochondria was recorded. Fluorescence intensity was calculated using Image-Pro Plus 6.0 software. First, fluorescence pictures (red and green fluorescence) were converted to the grayscale pictures with the help of Image-Pro Plus 6.0 software. Then, $\mathrm{red} /$ green fluorescence intensities were separately recorded as the grayscale intensity. Subsequently, relative grayscale intensity was expressed as a ratio to that of control group.

\section{Transfection}

Adenovirus-Mst1 was transfected into cells to perform the gain-of-function assay. The pCMV6-Kan/Neo Mst1 plasmids (1,247 bp; forward, 5'-CATGGTTAGTCTTGCATTGTGC-3' and reverse, 5'-GGTAGGTCTCCATTATCTTCCA-3') were obtained from OriGene Technologies, Inc. Then, $3.0 \mu \mathrm{g}$ of the above plasmids were transfected into $293 \mathrm{~T}$ cells $\left(2 \times 10^{4}\right.$ cells/well, National Infrastructure of Cell Line Resource) in DMEM with 10\% FBS using Lipofectamine ${ }^{\circledR} 2000$ (Invitrogen). After 48 hours, the supernatant was collected to obtain the Mst1 adenovirus (Ad-Mst1), which was transfected into AGS cells in Opti-MEM media supplemented with Lipofectamine ${ }^{\circledR} 2000$ according to the manufacturer's protocol. ${ }^{33}$ Transfection was carried out for 48 hours under $5 \% \mathrm{CO}_{2}$ at $37^{\circ} \mathrm{C}$. Then, Western blotting was performed to verify the transfection efficiency. Null adenovirus (Ad-ctrl) transfection was used as the negative control group.

\section{RNA isolation and qPCR}

Total RNA was extracted using the Trizol reagent (Invitrogen). Then, cDNA was transcribed with a One-step RT-PCR kit (TransGen Biotech Co., Ltd., Beijing, China) according to the manufacturer's instructions. ${ }^{24}$ qPCR was performed using the SYBR green PCR system on a 7500 fast (Applied Biosystem). The mRNA levels were normalized to the levels of GAPDH using the $2^{-\triangle \Delta C T}$ methods. ${ }^{34}$ The primer sequences used in the present study were as follows: Mst1, forward 5'-GCTGAGGAGCATGACAGACA-3' and reverse 5'-GATGAAGGCCAGGATGAGAA-3'. The cycling conditions were as follows: $95^{\circ} \mathrm{C}$ for 8 minutes, 350 cycles of $95^{\circ} \mathrm{C}$ for 15 seconds, and $72^{\circ} \mathrm{C}$ for 35 seconds.

\section{Data analysis}

All results presented in this study were acquired from at least three independent experiments unless indicated specifically. Data are presented as the mean \pm SEM. All statistical analyses were performed by one-way ANOVA followed by Dunnett's test or Tukey's test for comparing variable groups using GraphPad Prism 5 software. $P<0.05$ was considered statistically significant.

\section{Results \\ Overexpression of Mst I reduces gastric cancer cell viability}

Initially, qPCR was performed to analyze the expression of Mst1 in gastric cancer cells. As shown in Figure 1A, compared to the normal gastric mucosal cell line (GES-1 cells), the transcription of Mst1 was significantly downregulated in the gastric cancer cell line (AGS cells). This finding was validated by Western blotting, which demonstrated that lower levels of Mst1 were identified in AGS cells when compared with those in GES-1 cells (Figure 1B and C). This information indicates that Mst1 is downregulated in gastric cancer cells. Subsequently, Ad-Mst1 was transfected into AGS cells to overexpress Mst1. The transfection efficiency was verified via Western blotting which showed that 
A

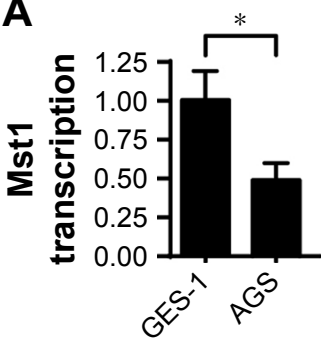

B

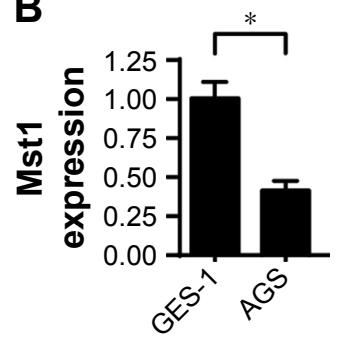

C

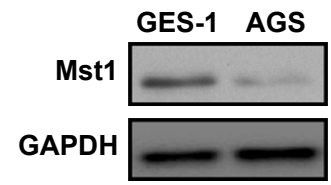

D

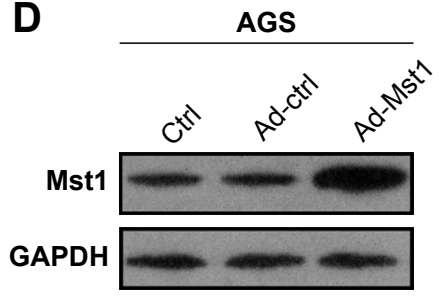

E

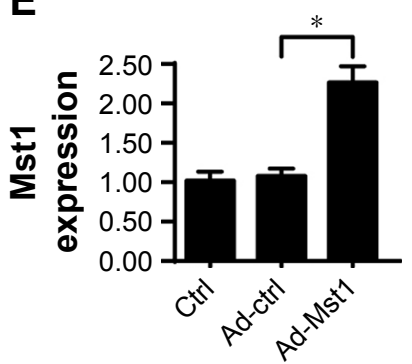

$\mathbf{F}$

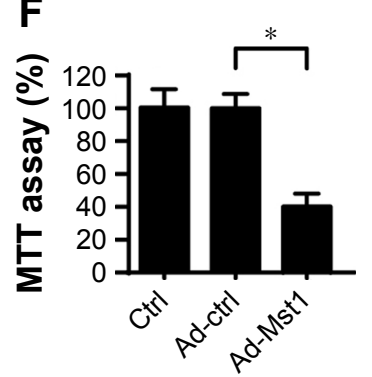

G

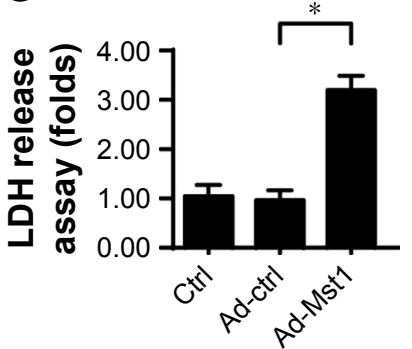

H

AGS

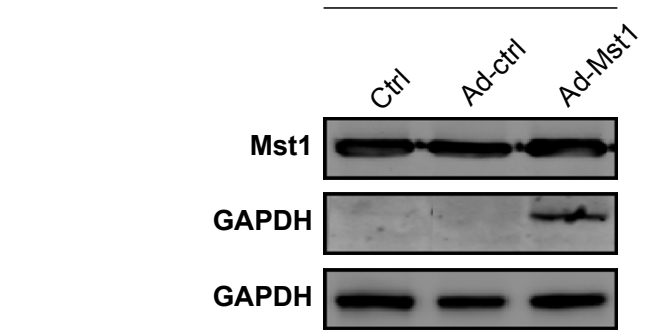

I
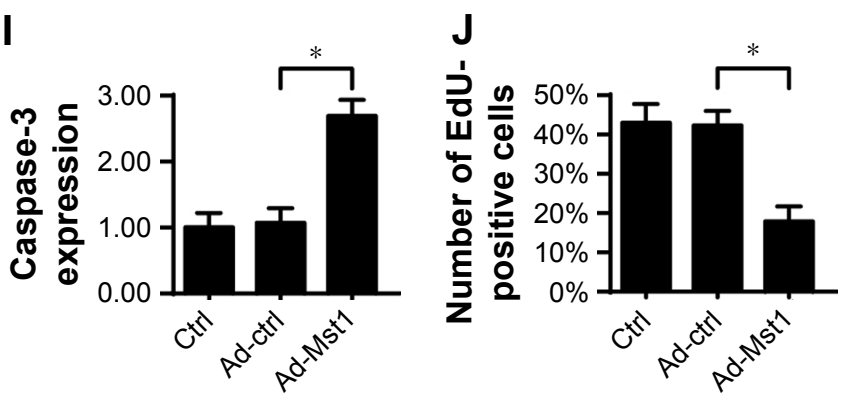

K

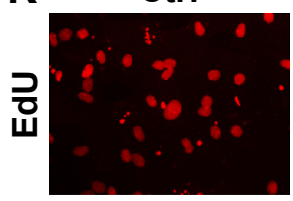

Ad-ctrl

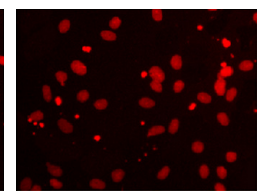

Ad-Mst1
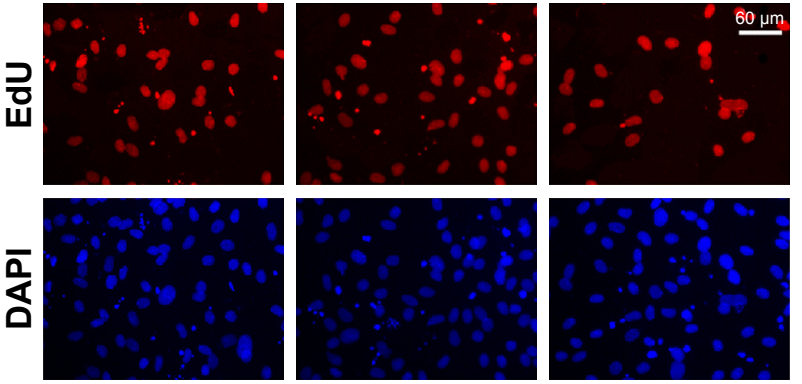

Figure I Mst I regulates gastric cancer cell viability

Notes: (A) qPCR assay for Mstl transcription in GES-I and AGS cells. (B, C) Western blotting assay for Mst I expression in GES-I and AGS cells. (D, E) To overexpress Mstl in AGS cells, adenovirus Mstl (Ad-Mstl) was transfected into AGS cells. The transfection was verified via Western blotting. (F) MTT assay was used to evaluate cell viability. Ad-Mst I was transfected into AGS cells. The null adenovirus was transfected as the control group (Ad-ctrl). (G) LDH release assay for cell death in AGS cells in response to Ad-Mst I transfection. (H, I) Caspase-3 activity was measured to reflect the activation of caspase proteins. (J, K) EdU assay for cell proliferation. The percentage of EdU-positive cells was recorded. Ad-MstI and/or Ad-ctrl were transfected into AGS cells. $* P<0.05$ vs control group.

Abbreviation: Mstl, mammalian sterile 20-like kinase I.

transfection of Ad-Mst1 drastically increased the levels of Mst1 in AGS cells when compared with the control group (Figure 1D and E). To observe the phenotypic alteration in response to Mst1 overexpression, MTT assay was conducted to measure cellular viability. Compared to the control group, Ad-Mst1 transfection significantly reduced cellular viability (Figure 1F). Moreover, the decreased cell viability may be attributed to increased cell death because the LDH release assay showed that Ad-Mst1 transfection promoted LDH release into the medium of AGS cells (Figure 1G). Similarly, caspase-3 expression was also elevated in response to AdMst1 (Figure 1H and I), confirming that Mst1 overexpression promoted gastric cancer death. In addition, we found that Mst1 overexpression statistically reduced the number of EdUpositive cells (Figure 1J and K), suggesting a Mst1-mediated cell proliferation arrest. Together, our data demonstrate that gastric cancer cells lack Mst1, and overexpression of Mst1 reduces tumor cell viability by promoting cell death. 


\section{Mst I mediates mitochondrial damage}

Mitochondria have been acknowledged as the potential target to control cell viability. ${ }^{35}$ Accordingly, we measured mitochondrial function with Mst1 overexpression. First, the total ATP production in the cell was monitored via ELISA. The results in Figure 2A demonstrated that the concentration of cell ATP was repressed in Mst1-overexpressed cells. Notably, the cellular ATP production is primarily regulated by mitochondria via the mitochondrial respiratory complex. Interestingly, Ad-Mst1 transfection led to a sharp decline in the levels of mitochondria respiratory complex (Figure 2B-E). ATP chemical energy mainly results from the mitochondrial membrane potential. Compared to the control group, Mst1 overexpression significantly reduced the mitochondrial membrane potential (Figure $2 \mathrm{~F}-\mathrm{G}$ ), as evidenced by decreased red-to-green fluorescence intensity.

As a consequence of mitochondrial membrane potential reduction, excessive electron diffusion into the cytoplasm would occur, contributing to the formation of ROS. ${ }^{36}$ The flow cytometry analysis of cellular ROS confirmed that Mst1 overexpression increased the content of ROS when compared with the control group (Figure $2 \mathrm{H}$ and I). In response to the ROS overproduction, the concentration of cellular antioxidants such as SOD, GSH and GPX was statistically downregulated in Mst1-overexpressed cells (Figure 2J-L), suggesting that Mst1 overexpression induced cancer oxidative stress. Altogether, these results demonstrated that activation of Mst1 resulted in mitochondrial malfunction in gastric cancer cells.

\section{Mst I activates caspase-9-related mitochondrial apoptosis}

Previous studies have reported that excessive mitochondrial damage would activate the mitochondria-dependent apoptotic pathway. ${ }^{37}$ The following experiments were performed to evaluate the contribution of Mst1 overexpression to mitochondrial apoptosis. First, Western blotting was performed to observe the apoptotic proteins related to mitochondrial death. Compared to the control group, the expression of Bad and caspase- 3 were significantly upregulated in response to Mst1 overexpression (Figure 3A-F). In contrast, the expression of Bcl-2 and c-IAP was drastically downregulated in Mst1-overexpressed cells (Figure 3A-F). Mitochondrial apoptosis is characterized by caspase- 9 activation. Interestingly, Mst1 overexpression elevated the expression (Figure 3A and C) and activity (Figure 3G) of caspase-9 in
AGS cells, highlighting the critical role of Mst1 in initiating the caspase-9 mitochondria apoptotic pathway.

To determine how Mst1 overexpression activated caspase-9 mitochondrial apoptosis, we focused on cyt-c translocation. Under physical conditions, cyt-c is located in the mitochondria where cyt-c promotes electron transfer. Once liberated into the cytoplasm, cyt-c interacts with and activates caspase-9, launching mitochondrial apoptosis. ${ }^{26}$ Based on this, immunofluorescence was performed to analyze the sub-cellular location of cyt-c. Compared to the control group, Mst1 overexpression induced cyt-c translocation into the cytoplasm and nucleus (Figure 3H and I). Notably, cyt-c translocation from mitochondria into cytoplasm was regulated by the opening of the mPTP. Compared to the control group, Ad-Mst1 transfection elevated the ratio of mPTP opening (Figure 3J). In summary, Mst1 overexpression promoted mPTP opening, facilitated cyt-c liberation, increased the levels of pro-apoptotic proteins, and activated the caspase9-related mitochondrial apoptosis signaling pathway.

\section{Increased Mstl is associated with elevated mitochondrial fission}

The following experiments were carried out to explore the mechanism by which Mst1 promoted mitochondrial apoptosis. Previous studies identify mitochondrial fission as the upstream regulator of mitochondrial function. ${ }^{21}$ We investigated whether mitochondrial fission is required for Mst1-initiated mitochondrial apoptosis. First, immunofluorescence of mitochondria was conducted using the mitochondrial-specific antibody Tom-20. As shown in Figure 4A, elongated mitochondria were seen in control cells. Interestingly, Ad-Mst1 transfection promoted mitochondria division into several fragments. Moreover, we measured the average length of the mitochondria to quantify mitochondrial fission. The mitochondrial length was $\sim 8.9 \mu \mathrm{m}$ in control cells and was reduced to $2.3 \mu \mathrm{m}$ in Mst1-overexpressed cells (Figure 4B). These results illustrated that mitochondrial fission was activated by Mst1 overexpression. Mitochondrial fissionrelated proteins were further examined via Western blotting. Compared to the control group, Mst1 overexpression increased the expression of Drp1, Mff and Fis1 (Figure 4C-I), the executors of mitochondrial fission. Notably, the content of mitochondrial fission inhibitors such as Mfn2, Opa1 and Parkin were significantly downregulated in Mst1overexpressed cells when compared with the control cells (Figure 4C-I). This information confirmed the supportive effects of Mst1 in mitochondrial fission. 


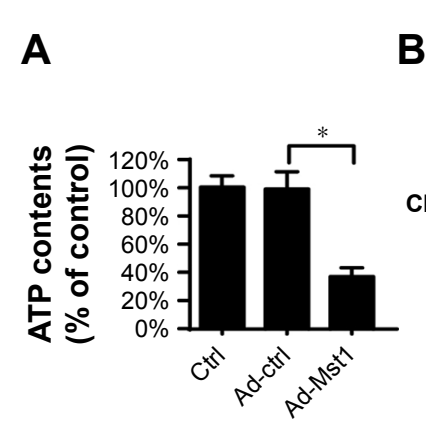

E

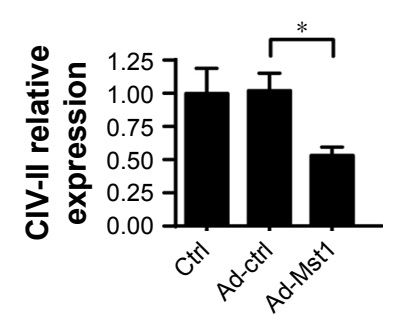

$\mathbf{F}$

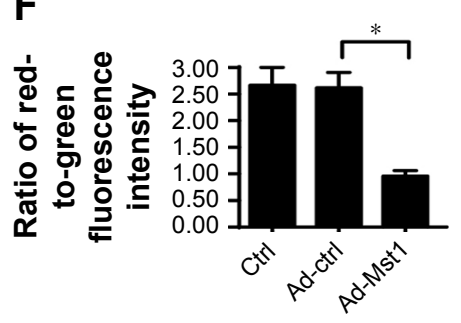

B

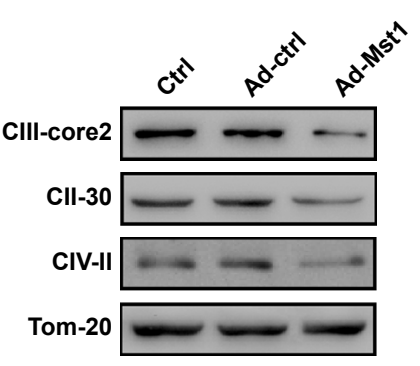

\section{C}

D

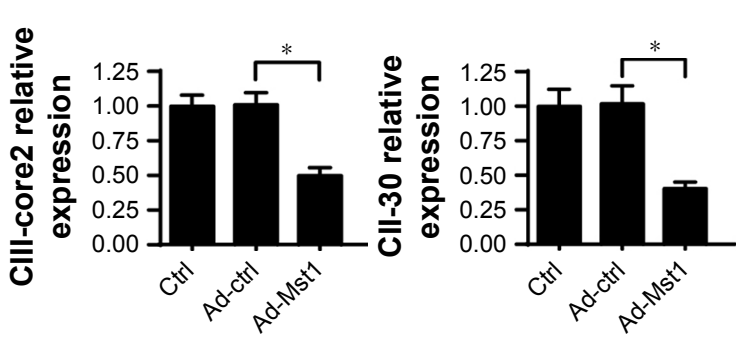

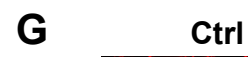
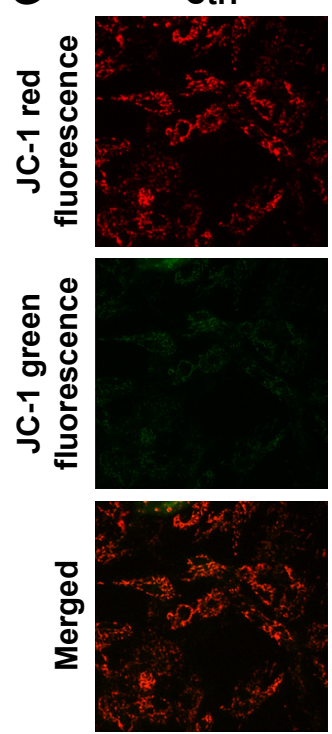

Ad-ctrl
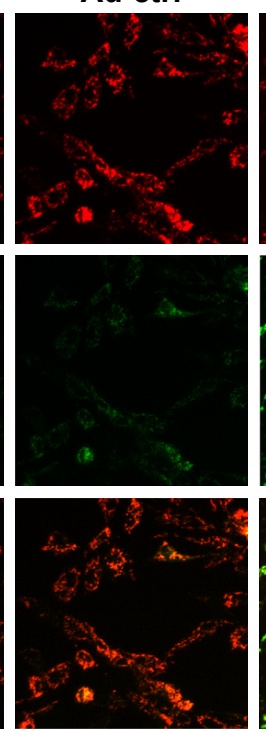

Ad-Mst1

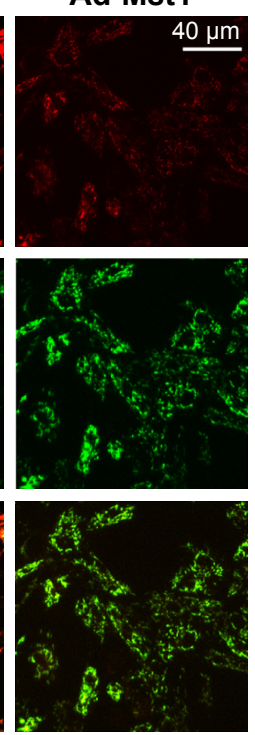

H
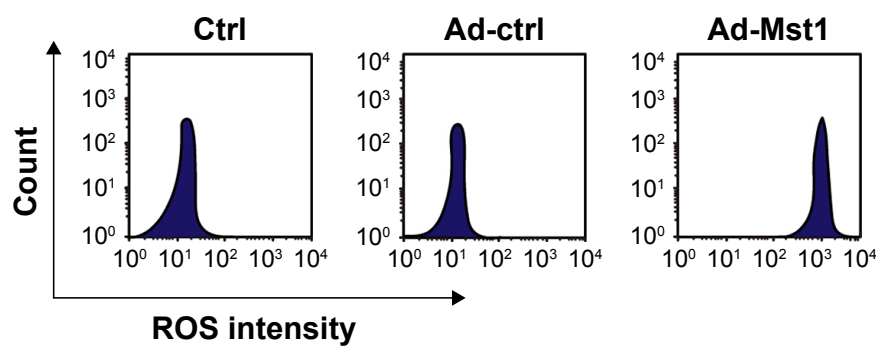

I

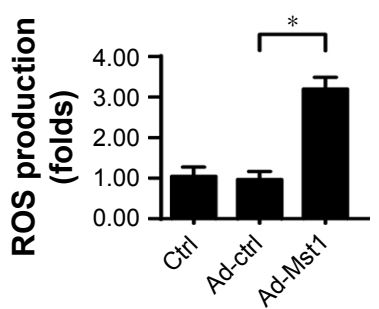

J

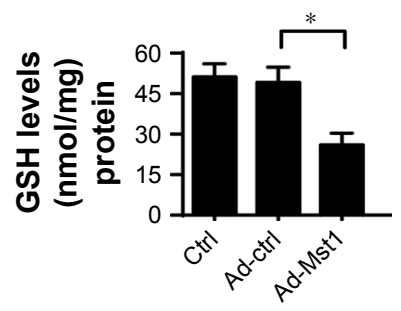

K

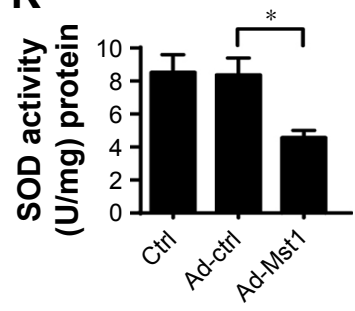

$\mathbf{L}$

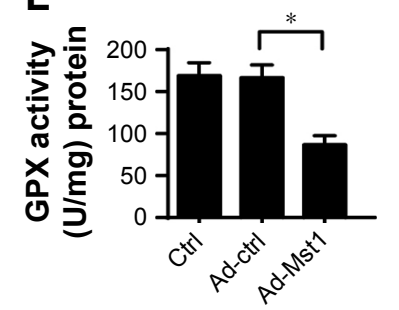

Figure 2 Overexpression of Mst l promotes mitochondrial damage.

Notes: (A) Cellular ATP content was evaluated to monitor the function of mitochondrial metabolism. Mstl adenovirus (Ad-Mstl) was transfected into AGS cells. The null adenovirus was transfected into the control group (Ad-ctrl). (B-E) The expression of the mitochondrial respiratory complex was quantified via Western blotting. Mst I overexpression repressed the levels of the mitochondrial respiratory complex. (F, G) JC-I staining for mitochondrial potential. Normal mitochondrial potential stained with the JC-I probe exhibits red fluorescence, whereas reduced mitochondrial potential tagged with the JC-I probe indicates green fluorescence. The red-to-green fluorescence ratio was calculated to reflect the alteration of mitochondrial potential. (H, I) Cellular ROS production was determined using the ROS probe. Quantification of cellular ROS was conducted via flow cytometry. (J-L) ELISA for SOD, GSH and GPX detection. The concentration of cellular antioxidants was measured via ELISA. $* P<0.05$ vs control group. Abbreviation: Mst I, mammalian sterile 20-like kinase I. 
A

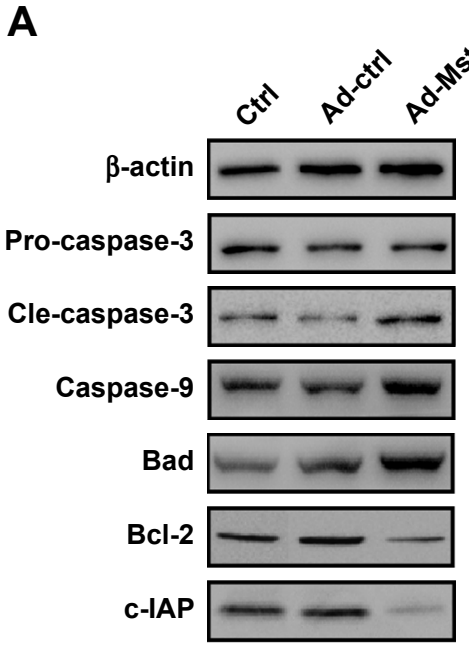

B

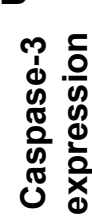

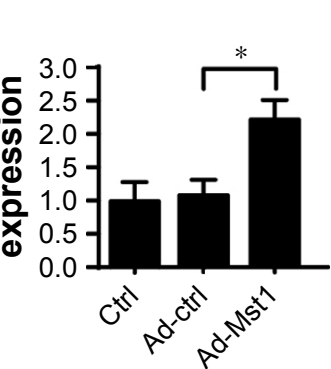

E

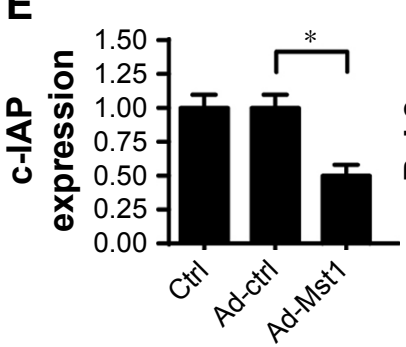

C

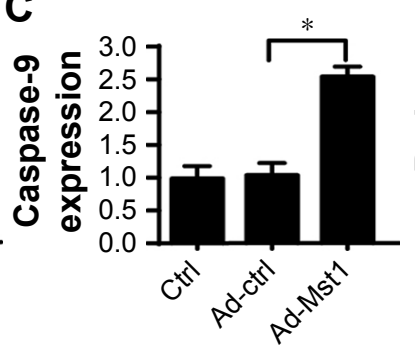

F

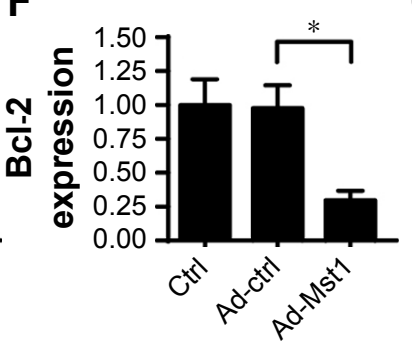

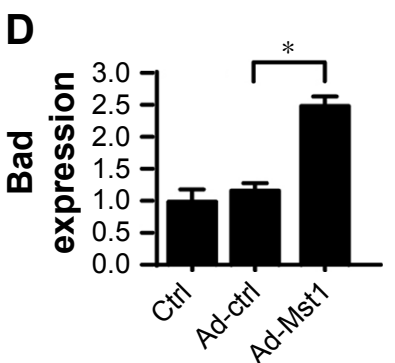

G

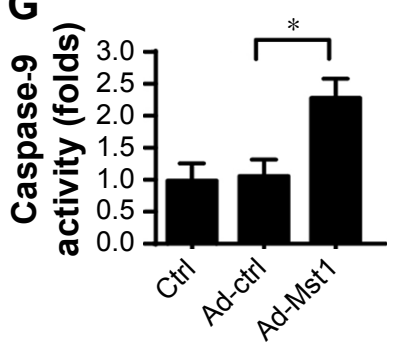

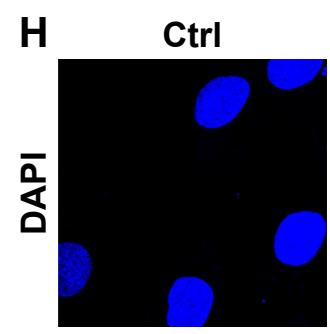
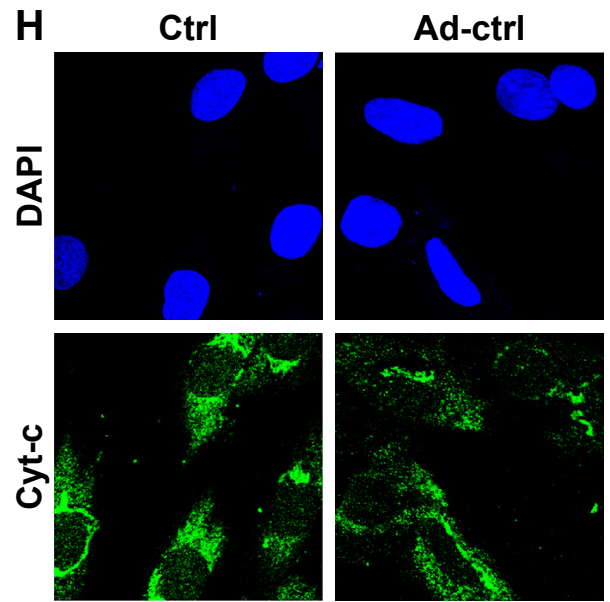

Ad-Mst1
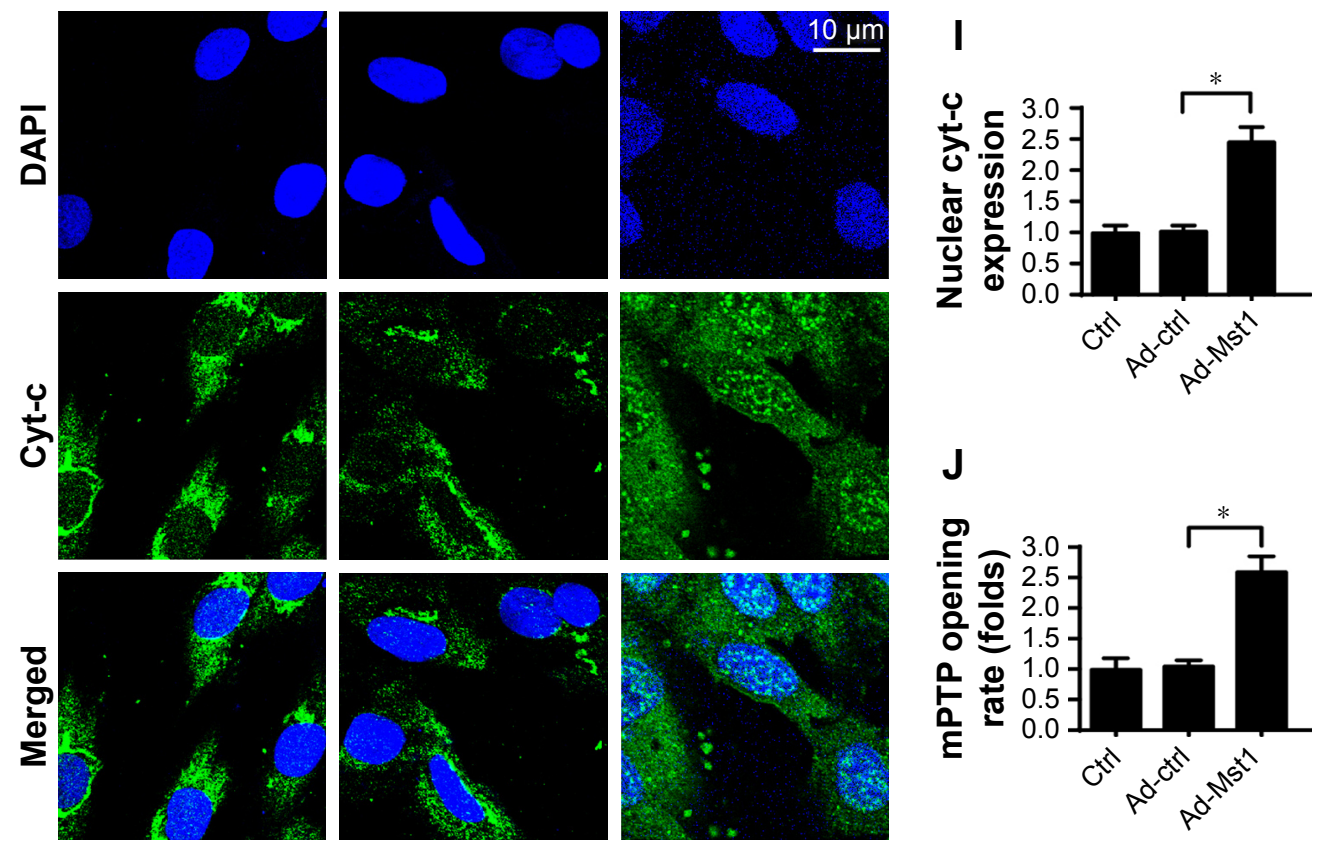

Figure 3 Caspase-9-dependent apoptotic pathway is activated by Mstl overexpression.

Notes: (A-F) The alterations of apoptosis-related proteins were measured via Western blotting. Caspase-3, Bad and caspase-9 were proapoptotic proteins the expression of which was upregulated by Mstl overexpression. In contrast, Bcl-2 and c-IAP were the antiapoptotic factors the levels of which were downregulated by Ad-Mstl transfection. (G) Caspase-9 activity was evaluated in response to Mstl overexpression. (H, I) Immunofluorescence assay for cyt-c. DAPI was used to label the nucleus, and the co-location of cyt-c, and DAPI indicated the translocation of mitochondrial cyt-c into the nucleus. (J) mPTP opening was determined in response to Ad-Mstl transfection. $* P<0.05$ vs control group.

Abbreviations: mPTP, mitochondrial permeability transition pore; Mst I, mammalian sterile 20-like kinase I.

\section{Mitochondrial fission is required for Mst I-mediated cell death via mitochondrial apoptosis}

To determine whether mitochondrial fission is necessary for Mst1-mediated cell death, loss- and gain-of-function assays for mitochondrial fission were performed. Mdivi-1, an antagonist of mitochondrial fission was supplemented into Mst1-overexpressed cells to inhibit mitochondrial fission.
In contrast, FCCP, an agonist was added into control cells to activate mitochondrial fission, which was then considered as the positive control group. Then, cell apoptosis was evaluated via TUNEL assay. Compared to the control group, Mst1 overexpression significantly increased the number of TUNEL-positive cells, and this effect was negated by Mdivi-1 (Figure 5A and B), suggesting that inhibition of mitochondrial fission abrogated the proapoptotic effects of 
A
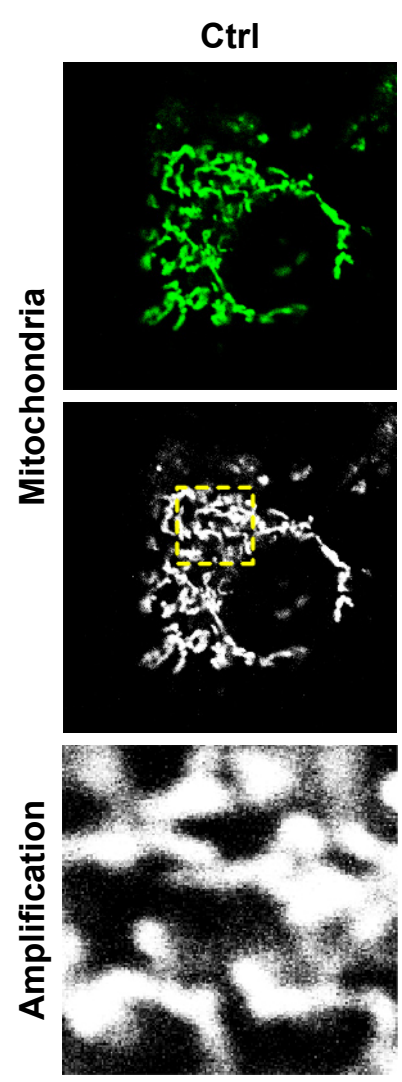

D

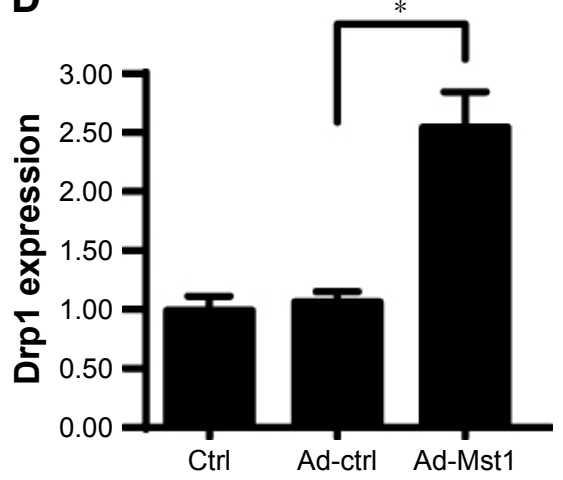

G

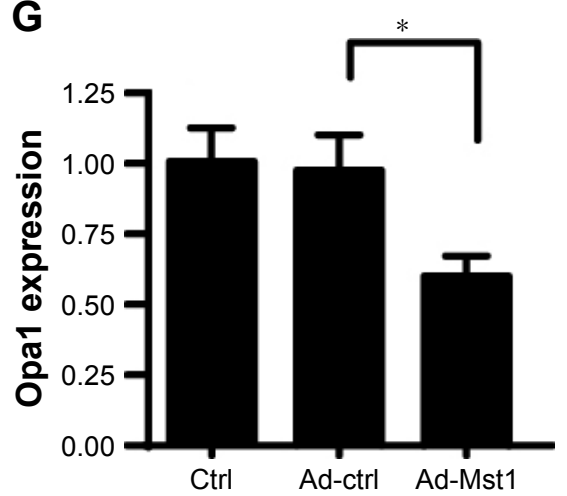

Ad-ctrl
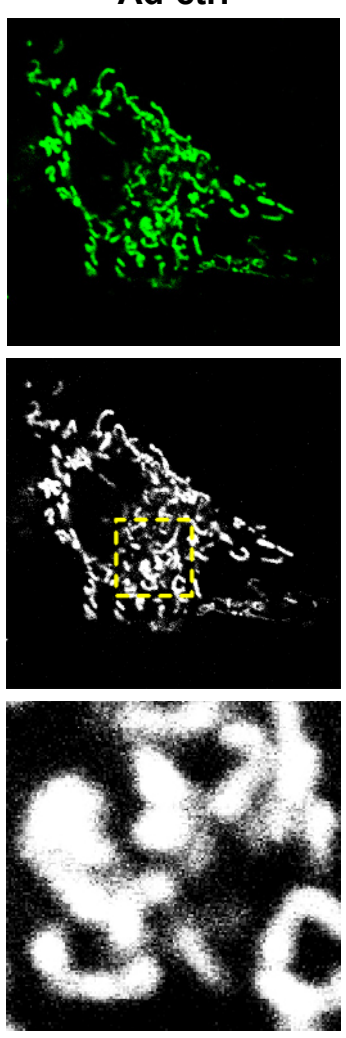

E

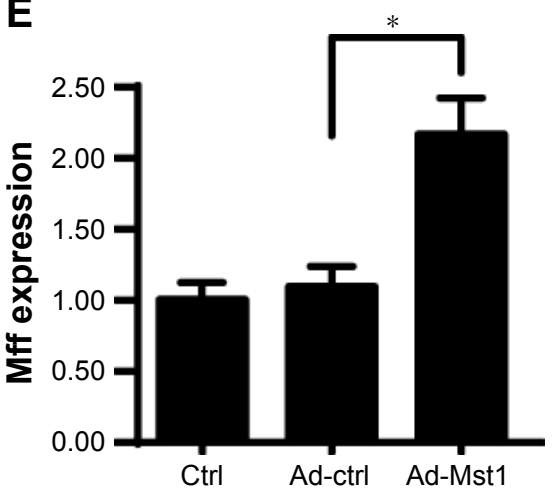

H

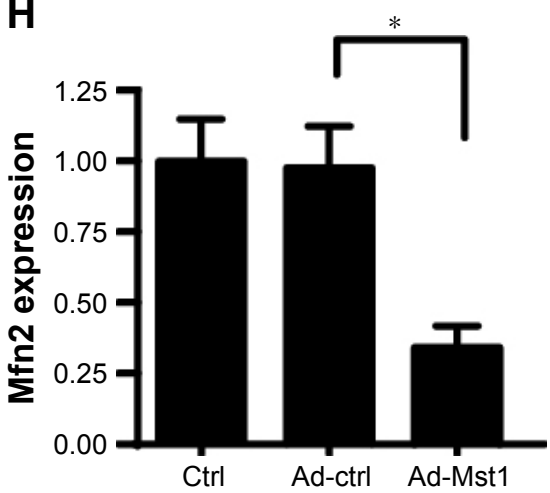

B

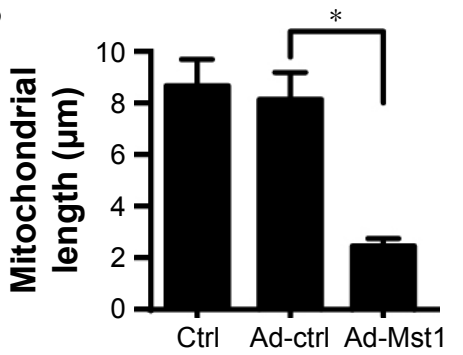

C
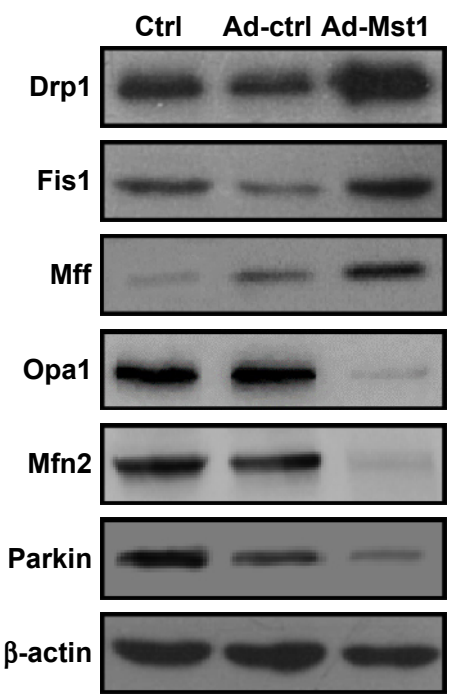

$\mathbf{F}$
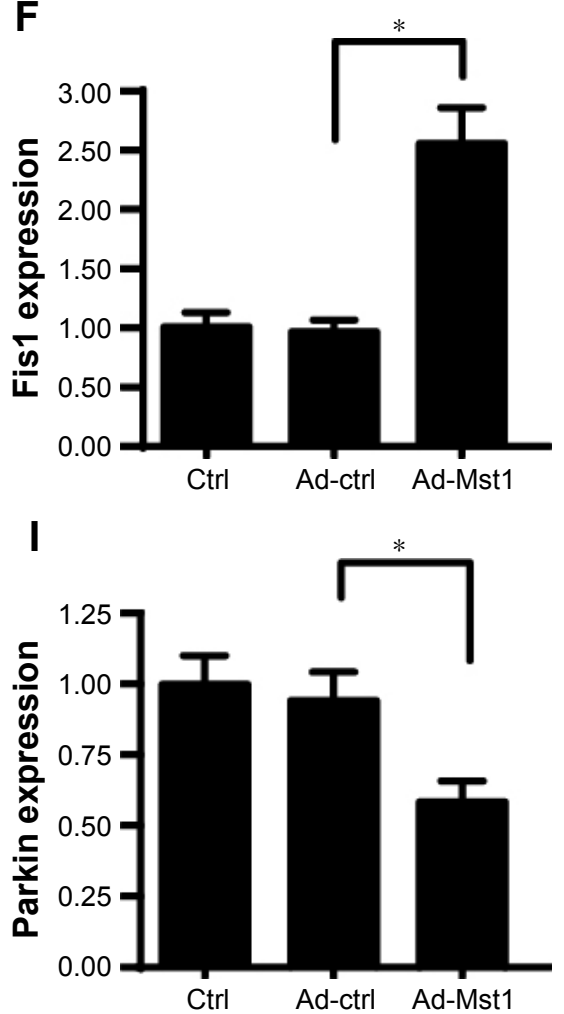

Figure $4 \mathrm{Mst}$ l overexpression activates mitochondrial fission.

Notes: (A) Mitochondrial immunofluorescence was performed using the mitochondrial-specific antibody Tom-20. Then, the amplification of mitochondria was recorded. (B) The average mitochondrial length was measured, which quantifies mitochondrial fission. (C-I) Mitochondrial fission-related factors were determined via Western blotting. Drpl, Mff and Fis I were the mitochondrial fission activators, and their expression was increased by Mstl overexpression. Mfn2, Parkin and Opal were the mitochondrial fission inhibitors, and their levels were repressed by Mst I overexpression. $* P<0.05$ vs control group.

Abbreviation: Mstl, mammalian sterile 20-like kinase I. 

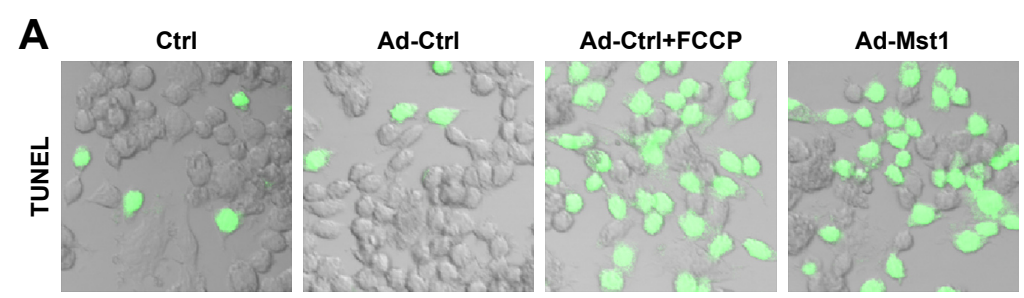

Ad-Mst1+Mdivi-1
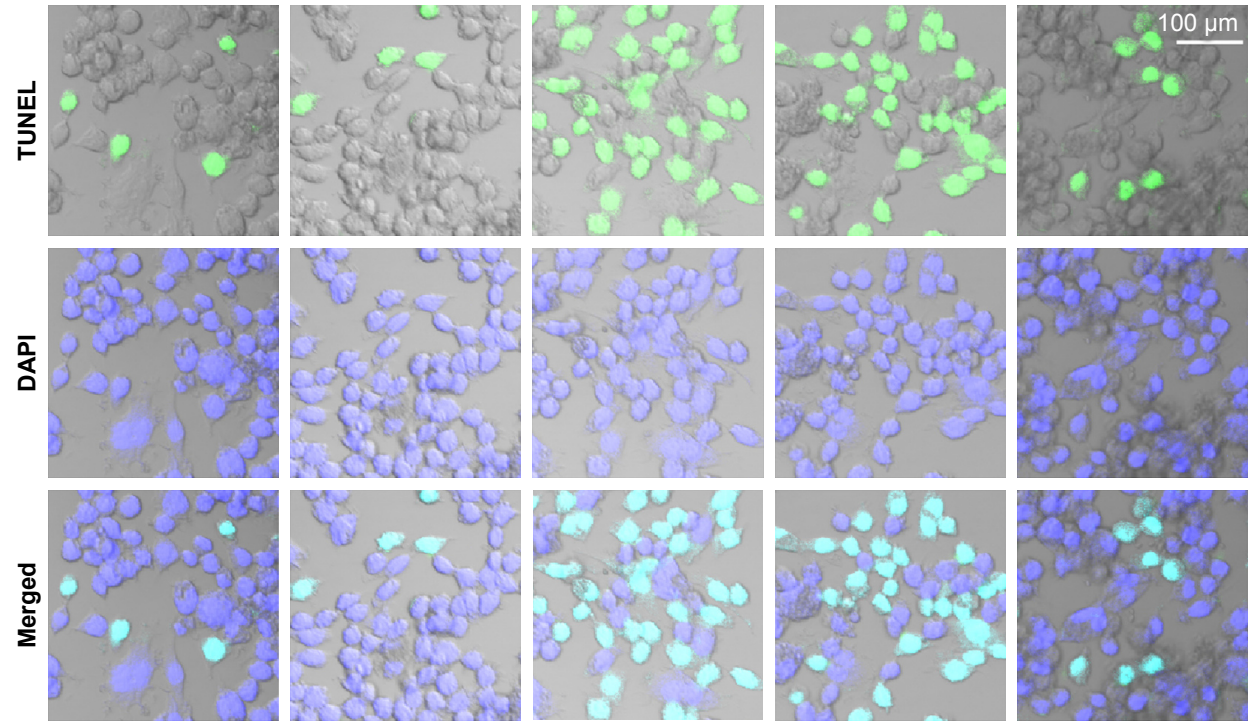

B

C

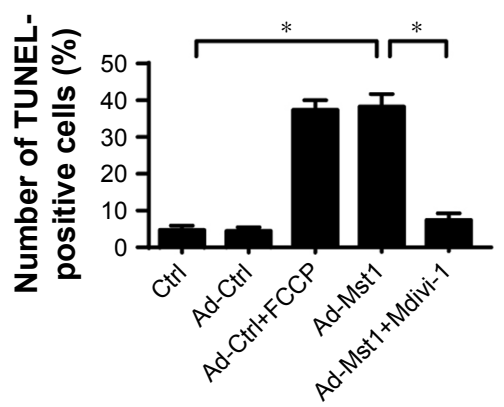

E

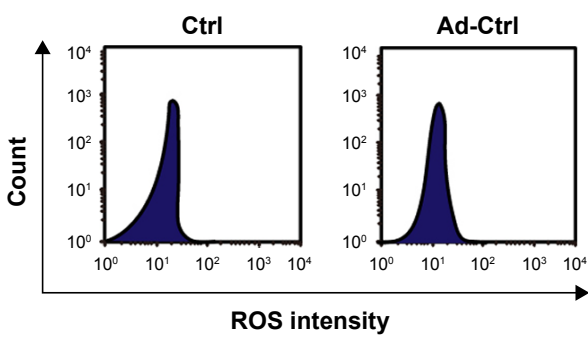

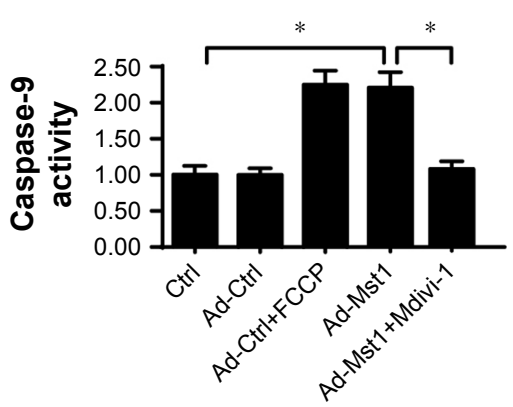

D
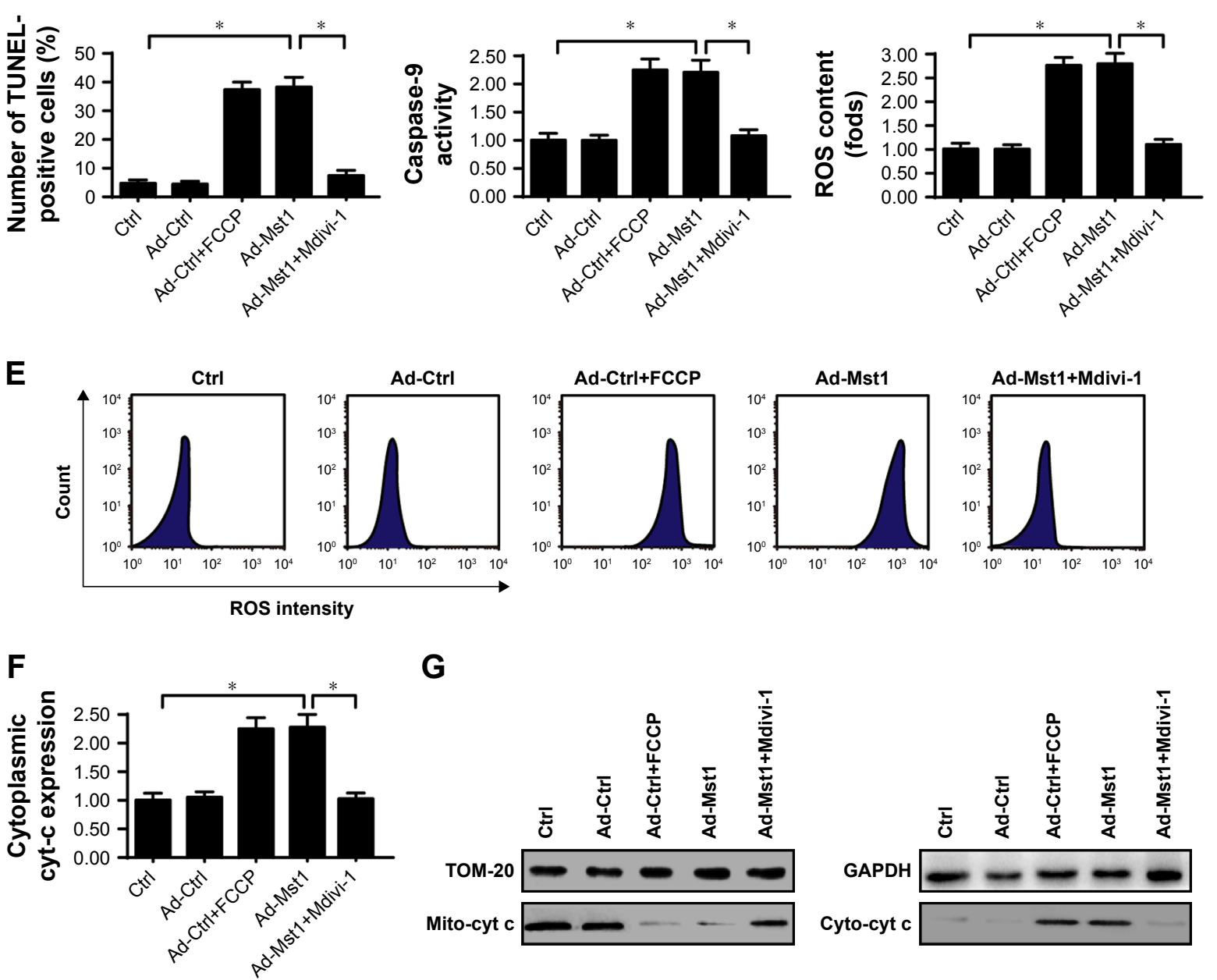

G

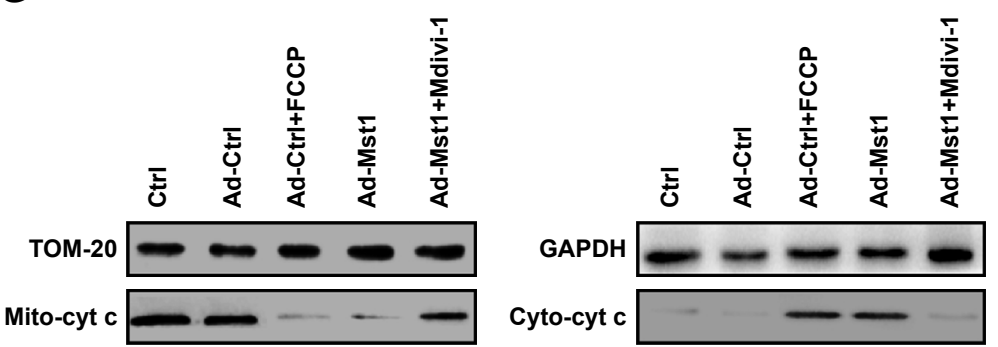

Figure $5 \mathrm{Mst}$-activated mitochondrial fission accounts for mitochondrial apoptosis.

Notes: (A, B) TUNEL staining for apoptotic cells. The ratio of TUNEL-positive cells was recorded. Meanwhile, FCCP and Mdivi-I, the agonist and antagonist for mitochondrial fission, respectively, were used to conduct the gain- and loss-of-function assays for mitochondrial fission. In Mst I-overexpressed cells, Mdivi-I was added to inhibit mitochondrial fission. In cells transfected with Ad-ctrl, FCCP was added to activate mitochondrial fission. (C) Caspase-9 activity was measured in response to Mst I overexpression and/or mitochondrial fission inhibition. (D, E) Cellular ROS production was measured via flow cytometry with mitochondrial fission inhibition and/or Mst I overexpression. (F, G) The mitochondrial cyt-c translocation assay was estimated via immunofluorescence. Nucleus was labeled by DAPI. *P<0.05 vs control group. Abbreviation: Mst I, mammalian sterile 20-like kinase I. 
Mst1 overexpression on gastric cancer cells. Moreover, the caspase-9 activity was drastically increased in response to Mst1 overexpression (Figure 5C); this effect, which was nullified by Mdivi-1 administration (Figure 5C), confirms that Mst1initiated cell death is dependent on mitochondrial fission.

Regarding mitochondrial dysfunction, ROS production and cyt-c translocation were assessed again. As shown in Figure 5D and E, compared to the control group, ROS production was significantly augmented by Mst1 overexpression or FCCP treatment. By comparison, Mdivi-1 administration repressed ROS production in Mst1-overexpressed cells (Figure 5D and E). Similarly, Mst1 promoted cyt-c translocation into the cytoplasm (Figure 5F and G), and this effect was mostly reversed by Mdivi-1. Altogether, the above data indicated that Mst1 regulated mitochondrial function and gastric cancer cell death via mitochondrial fission.
Mstl regulates mitochondrial fission via the AMPK-Sirt3 pathway

Finally, we investigated the molecular mechanism by which Mst1 modulated mitochondrial fission in gastric cancer cells. According to previous reports, mitochondrial fission is primarily regulated by two signaling pathways. The AMPK pathway is the upstream inhibitory mechanism for mitochondrial fission, ${ }^{38}$ and the MAPK-JNK cascade is the initial signal for mitochondrial fission. ${ }^{21}$ Moreover, as the downstream mediator of the AMPK pathway, Sirt3 has been known as the defender of mitochondrial homeostasis. ${ }^{21}$ Higher Sirt3 expression suppresses excessive mitochondrial fission. In the present study, we explored the role of the AMPK-Sirt3 pathway in Mst1-activated mitochondrial fission. Western blotting assays demonstrated that abundant p-AMPK was seen in the control cells (Figure 6A and B). However, Mst1

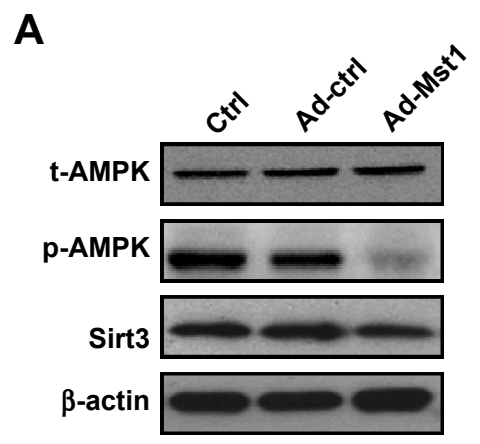

B
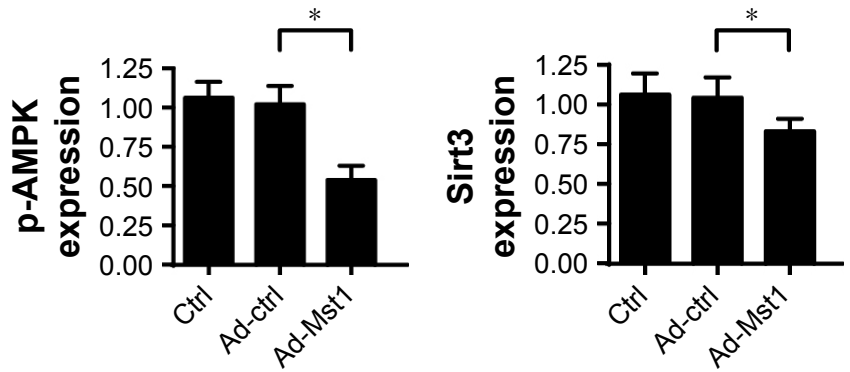

C
D
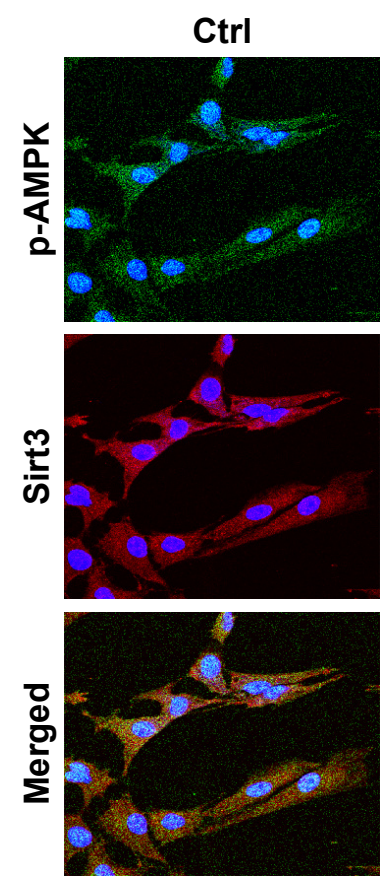

Ad-ctrl
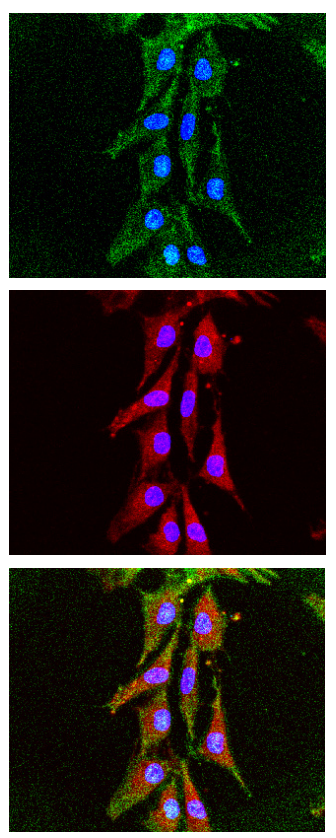

Ad-ctrl + compound C
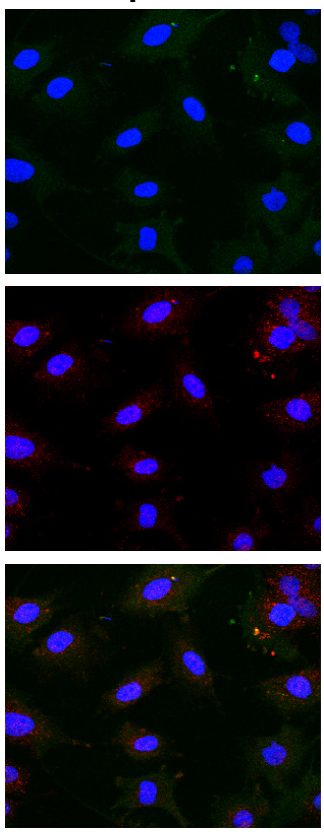

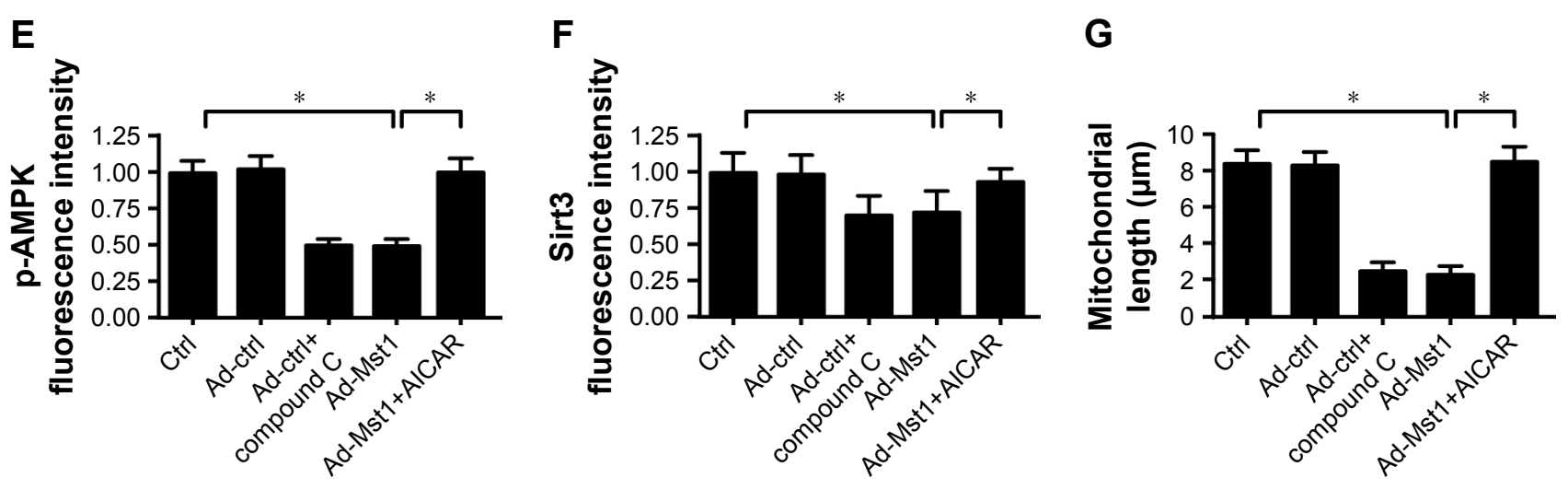

H
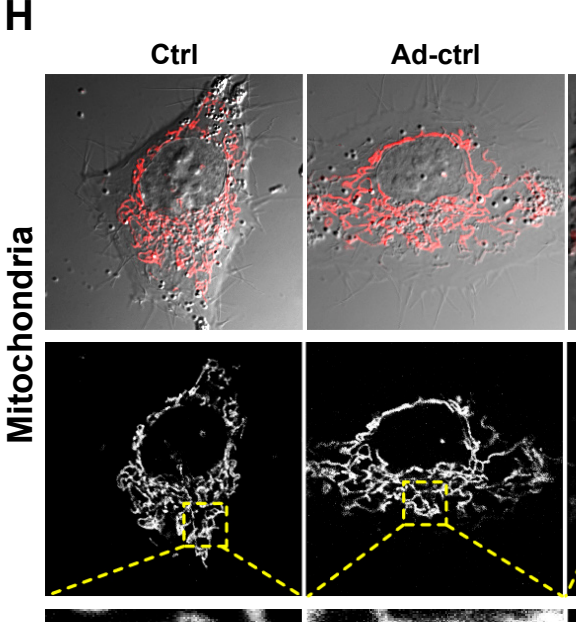

\section{Ad-ctrl+}
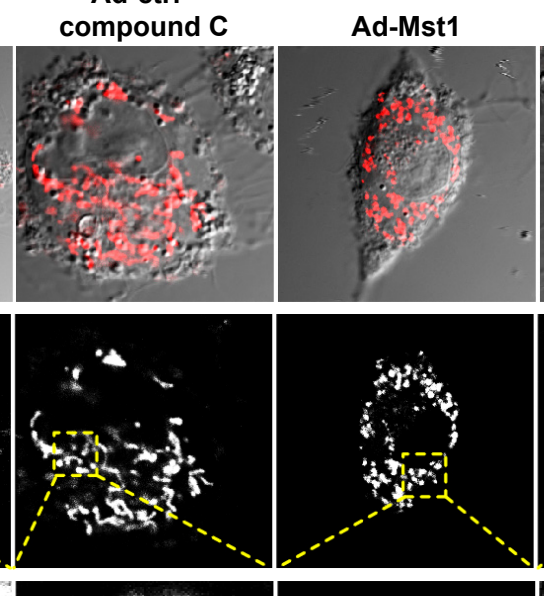

Ad-Mst1+AICAR
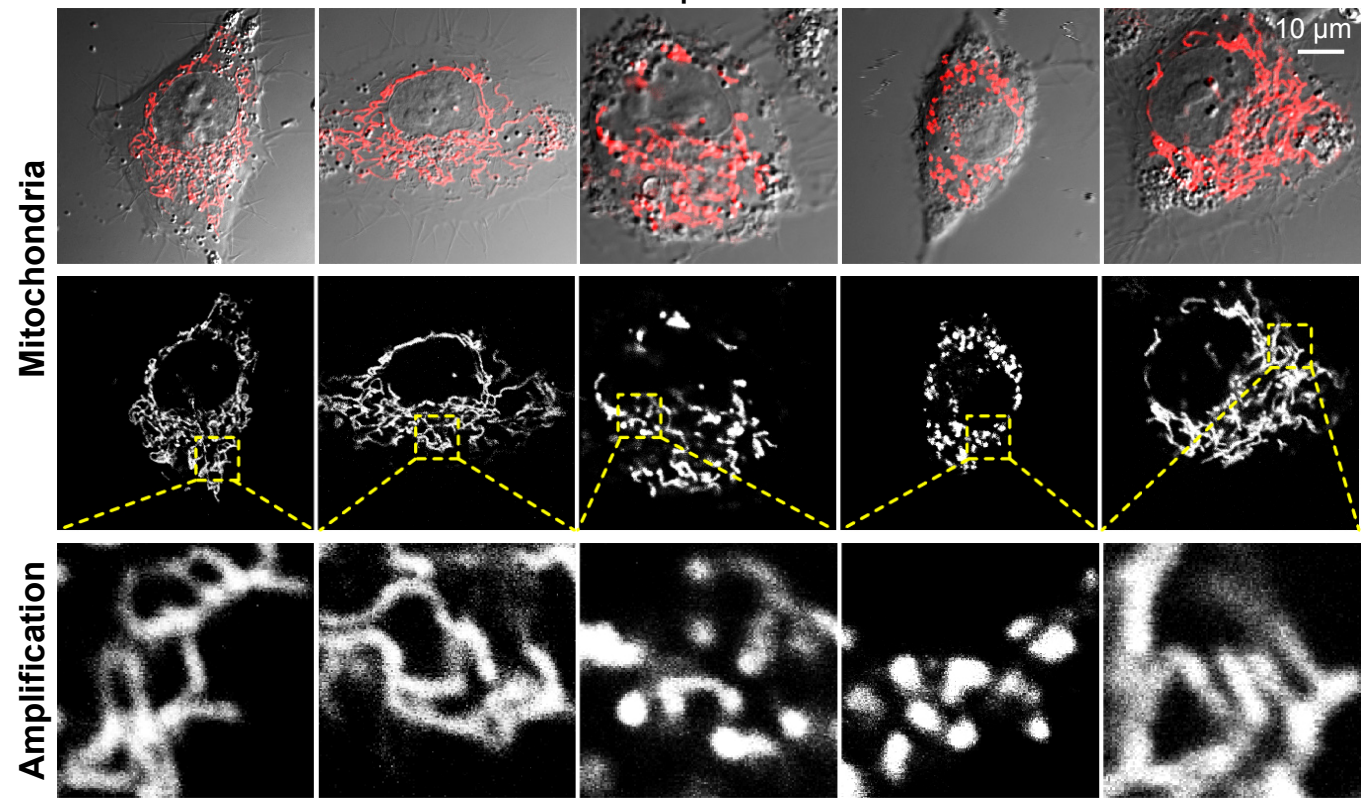

Figure 6 Mstl modulates mitochondrial fission via AMPK-Sirt3 pathways.

Notes: (A-C) The activation of the AMPK-Sirt3 pathway was evaluated via Western blotting. AMPK phosphorylation and Sirt3 expression were recorded in response to Mst I overexpression. (D-F) Immunofluorescence of P-AMPK and Sirt3 in response to Mst I overexpression. Meanwhile, the agonist and antagonist of the AMPK-Sirt3 pathway were added using AICAR (AI) and Compound C (CC), respectively. In Mst I-overexpressed cells, Al was added to re-activate AMPK phosphorylation and Sirt3 expression. In cells transfected with Ad-ctrl, CC was administered to inhibit the AMPK-Sirt3 pathways, which was used to mimic the effects of Mst I overexpression. (G, H) Mitochondrial fission was re-evaluated using immunofluorescence. The average length of mitochondria was recorded to quantify mitochondrial fission. In Mst I-overexpressed cells, Al was added to re-activate AMPK phosphorylation and Sirt3 expression. In cells transfected with Ad-ctrl, CC was administered to inhibit the AMPK-Sirt3 pathways, which was used to mimic the effects of Mstl overexpression. $* P<0.05$ vs control group.

Abbreviation: Mst I, mammalian sterile 20-like kinase I.

overexpression significantly repressed the expression of p-AMPK in gastric cancer cells (Figure 6A and B), indicative of AMPK inactivation with Mst1 overexpression. Moreover, Sirt3 expression was also downregulated in response to Ad-Mst1 transfection (Figure 6A-C), supporting the inhibitory effects of Mst1 on Sirt3 expression. This finding was validated via immunofluorescence (Figure 6D-F). The fluorescence intensity of p-AMPK and Sirt3 were both largely repressed by Mst1 overexpression (Figure 6D-F). To verify the functional role of the AMPK-Sirt3 pathway in Mst1-initiated mitochondrial fission, loss- and gain-offunction assays for the AMPK pathway were performed. AI, the activator of the AMPK pathway was added into Mst1-overexpressed cells to re-activate the AMPK-Sirt3 pathway. In contrast, Compound $C$, the blocker of the AMPK pathway was administered into control cells to mimic the role of Mst1 overexpression. Then, mitochondrial fission was measured via immunofluorescence. As illustrated in Figure $6 \mathrm{G}$ and $\mathrm{H}$, Mst1 overexpression promoted the formation of mitochondrial fragments, which exhibited shorter 
length, and this effect was negated by AI. Collectively, these data confirmed that Mst1 modulated mitochondrial fission and gastric cancer cell death via the AMPK-Sirt3 pathway.

\section{Discussion}

In the present study, we identified Mst1 as a novel tumorsuppressive factor promoting cell death in gastric cancer. Our data suggested that Mst1 was downregulated in gastric cancer cells when compared with normal gastric mucosal cells. Interestingly, overexpression of Mst1 in gastric cancer cells reduced cellular viability by promoting cell apoptosis. Functional studies demonstrated that Mst1 overexpression induced mitochondrial damage by dissipating mitochondrial membrane potential, increasing ROS production, and decreasing antioxidants. In addition, damaged mitochondria in response to Mst1 overexpression also liberated cyt-c into the cytoplasm and nucleus to activate caspase-9, which amplified mitochondria-dependent apoptotic signaling in gastric cancer cells. These results uncovered the proapoptotic action of Mst1 on cancer cells. In accordance with our findings, previous studies have also reported the fatal effects of Mst1 in several kinds of cells. In cardiac post-infarction injury, Mst1 activation is associated with increased cardiomyocytes death, which contributes to heart remodeling. ${ }^{39}$ Moreover, Mst1 expression is coupled with liver cell death by inducing mitochondrial malfunction. ${ }^{40}$ In cancer cells, Mst1 regulates cell death in colorectal cancer, ${ }^{41}$ pancreatic cancer, ${ }^{42}$ hepatocellular carcinoma, ${ }^{43}$ breast cancer, ${ }^{3}$ esophageal cancer, ${ }^{44}$ and myeloid leukemia. ${ }^{45}$ Notably, our study is the first investigation to establish the lethal effect of Mst1 in gastric cancer cells. Our findings, combined with previous reports, confirm the central role of Mst1 activation that leads to apoptosis in various kinds of tumors. The increase of Mst1 expression could be of utmost importance when designing anti-cancer therapies in the clinical setting. Notably, the role of Mst1 in the normal tissue should be evaluated in the future.

At the molecular level, our data suggest that Mst1 activation regulates mitochondrial homeostasis via mitochondrial fission. Inhibition of mitochondrial fission preserved mitochondrial integrity and blocked the activation of mitochondrial apoptosis. ${ }^{21}$ In fact, mitochondrial fission has been identified to be an early event in mitochondrial damage. Two mechanisms involved in how mitochondrial fission induces mitochondrial damage and cell apoptosis stress have been reported; one is driven by ATP shortage, and the other involves caspase- 9 activation. Mechanistically, mitochondrial fission produces non-functional mitochondrial debris, which cannot perform oxidative phosphorylation, leading to ATP depletion. ${ }^{12}$ In addition, non-functional mitochondrial fragments with increased mitochondrial membrane permeability irreversibly promote cyt-c translocation into the cytoplasm and nucleus where cyt-c activates caspase- 9 and initiates the mitochondrial apoptosis program. ${ }^{46}$ These molecular mechanisms have been noted in different disease models such as myocardial ischemia-reperfusion injury, ${ }^{21}$ chronic fatty liver disease, ${ }^{22}$ endothelial oxidative stress, ${ }^{47}$ and neurodegeneration. ${ }^{48}$ In the present study, gastric cancer cell death was largely regulated by mitochondrial fission via Mst1, which provides a potential target to treat the development and progression of stomach tumors.

Finally, we explored the detailed mechanism involved in how Mst1 managed mitochondrial fission. We found that Mst1 activation repressed the AMPK-Sirt3 pathway and reactivation of the AMPK pathway abrogated the promoting effect of Mst1 on mitochondrial fission. On the basis of the previous studies, two signaling pathways have been recognized as the primary upstream regulators for mitochondrial fission. One is the AMPK pathway, and the other is the MAPK-JNK axis; the former is the inhibitory signal for mitochondrial fission, ${ }^{38}$ whereas the latter plays a decisive role in activating mitochondrial fission. ${ }^{49}$ In the self-renewal of human acute myeloid leukemia, ${ }^{50}$ the AMPK pathway represses mitochondrial fission and consequently maintains stem cell properties. Similarly, in a model of mouse diabetes, the AMPK pathway promotes the survival of tubular epithelium by correcting excessive mitochondrial fission. ${ }^{51}$ With respect to the MAPKJNK pathway, in-depth studies have uncovered the cross-talk between the MAPK-JNK axis and mitochondrial fission in models of liver cancer metastasis, post-infarction cardiac injury, ${ }^{52}$ endometriosis, rectal cancer, ${ }^{12}$ and microvascular ischemia-reperfusion insult. ${ }^{21}$ In the present study, we found that Mst1 overexpression inactivated the AMPK-Sirt3 pathway, which was accompanied by an increase in mitochondrial fission. These results help us understand the mechanism by which Mst1 regulates mitochondrial fission. Whether Mst1 performs mitochondrial fission via the MAPK-JNK pathway is not entirely understood, and further research is required.

Altogether, our results identified Mst1 as a new tumorsuppressive factor reducing gastric cancer survival. Increased Mst1 repressed the AMPK-Sirt3 pathway and enhanced fatal mitochondrial fission. Excessive mitochondrial division caused mitochondrial dysfunction and cell apoptosis. These findings highlight the role of mitochondrial fission as 
a master regulator of cancer cell viability. However, our data offer a new target for treating gastric cancer by regulating the AMPK-Sirt3 pathway.

\section{Data sharing statement}

The datasets used and/or analyzed during the current study are available from the corresponding author on reasonable request.

\section{Disclosure}

The authors report no conflicts of interest in this work.

\section{References}

1. Fornaro L, Vasile E, Aprile G, et al. Locally advanced gastro-oesophageal cancer: Recent therapeutic advances and research directions. Cancer Treat Rev. 2018;69:90-100.

2. Giampieri R, del Prete M, Cantini L, et al. Optimal management of resected gastric cancer. Cancer Manag Res. 2018;10:1605-1618.

3. Lin XY, Cai FF, Wang MH, et al. Mammalian sterile 20-like kinase 1 expression and its prognostic significance in patients with breast cancer. Oncol Lett. 2017;14(5):5457-5463.

4. Zhang M, Lin J, Wang S, et al. Melatonin protects against diabetic cardiomyopathy through Mst1/Sirt3 signaling. J Pineal Res. 2017;63(2): e12418.

5. Ikeda S, Sadoshima J. Regulation of myocardial cell growth and death by the Hippo pathway. Circ J. 2016;80(7):1511-1519.

6. Li X, Zhao X, Song W, et al. Pseudolaric acid B inhibits proliferation, invasion and epithelial-to-mesenchymal transition in human pancreatic cancer cell. Yonsei Med J. 2018;59(1):20-27.

7. García-Ruiz JM, Galán-Arriola C, Fernández-Jiménez R, et al. Bloodless reperfusion with the oxygen carrier HBOC-201 in acute myocardial infarction: a novel platform for cardioprotective probes delivery. Basic Res Cardiol. 2017;112(2):17.

8. Guo Z, Li G, Bian E, Ma CC, Wan J, Zhao B. TGF- $\beta$-mediated repression of MST1 by DNMT1 promotes glioma malignancy. Biomed Pharmacother. 2017;94:774-780.

9. Hong H, Tao T, Chen S, et al. MicroRNA-143 promotes cardiac ischemia-mediated mitochondrial impairment by the inhibition of protein kinase Cepsilon. Basic Res Cardiol. 2017;112(6):60.

10. Jovancevic N, Dendorfer A, Matzkies M, et al. Medium-chain fatty acids modulate myocardial function via a cardiac odorant receptor. Basic Res Cardiol. 2017;112(2):13.

11. Li Q, Qi F, Meng X, Zhu C, Gao Y. Mst1 regulates colorectal cancer stress response via inhibiting Bnip3-related mitophagy by activation of JNK/p53 pathway. Cell Biol Toxicol. 2018;34(4):263-277.

12. Li H, He F, Zhao X, et al. YAP inhibits the apoptosis and migration of human rectal cancer cells via suppression of JNK-Drp1mitochondrial fission-HtrA2/Omi pathways. Cell Physiol Biochem. 2017;44(5):2073-2089.

13. Chen L, Liu L, Li Y, Gao J. Melatonin increases human cervical cancer HeLa cells apoptosis induced by cisplatin via inhibition of JNK/Parkin/ mitophagy axis. In vitro Cell Dev Biol Anim. 2018;54(1):1-10.

14. Zhou H, Wang S, Hu S, Chen Y, Ren J. ER-mitochondria microdomains in cardiac ischemia-reperfusion injury: a fresh perspective. Front Physiol. 2018;9:755.

15. Zhou H, Wang J, Zhu P, et al. NR4A1 aggravates the cardiac microvascular ischemia reperfusion injury through suppressing FUNDC1mediated mitophagy and promoting Mff-required mitochondrial fission by CK2 $\alpha$. Basic Res Cardiol. 2018;113(4):23.

16. Zhou H, Shi C, Hu S, Zhu H, Ren J, Chen Y. BI1 is associated with microvascular protection in cardiac ischemia reperfusion injury via repressing Syk-Nox2-Drp1-mitochondrial fission pathways. Angiogenesis. 2018;21(3):599-615.
17. Archer SL. Mitochondrial dynamics - mitochondrial fission and fusion in human diseases. $N$ Engl J Med. 2013;369(23):2236-2251.

18. Nauta TD, van den Broek M, Gibbs S, et al. Identification of HIF-2 $\alpha-$ regulated genes that play a role in human microvascular endothelial sprouting during prolonged hypoxia in vitro. Angiogenesis. 2017;20(1): $39-54$.

19. Lassen TR, Nielsen JM, Johnsen J, Ringgaard S, Bøtker HE, Kristiansen SB. Effect of paroxetine on left ventricular remodeling in an in vivo rat model of myocardial infarction. Basic Res Cardiol. 2017; 112(3):26.

20. Zhou H, Wang S, Zhu P, Hu S, Chen Y, Ren J. Empagliflozin rescues diabetic myocardial microvascular injury via AMPK-mediated inhibition of mitochondrial fission. Redox Biol. 2018;15:335-346.

21. Jin Q, Li R, Hu N, et al. DUSP1 alleviates cardiac ischemia/reperfusion injury by suppressing the Mff-required mitochondrial fission and Bnip3-related mitophagy via the JNK pathways. Redox Biol. 2018;14:576-587.

22. Zhou H, du W, Li Y, et al. Effects of melatonin on fatty liver disease: the role of NR4A1/DNA-PKcs/p53 pathway, mitochondrial fission, and mitophagy. J Pineal Res. 2018;64(1):e12450.

23. Zhao H, Luo Y, Chen L, et al. Sirt3 inhibits cerebral ischemia-reperfusion injury through normalizing $\mathrm{Wnt} / \beta$-catenin pathway and blocking mitochondrial fission. Cell Stress Chaperones. 2018;23(5):1079-1092.

24. Zhou H, Li D, Zhu P, et al. Inhibitory effect of melatonin on necroptosis via repressing the Ripk3-PGAM5-CypD-mPTP pathway attenuates cardiac microvascular ischemia-reperfusion injury.J Pineal Res. 2018; 65(3): 12503.

25. Ren XS, Tong Y, Ling L, et al. NLRP3 gene deletion attenuates angiotensin II-induced phenotypic transformation of vascular smooth muscle cells and vascular remodeling. Cell Physiol Biochem. 2017;44(6):2269-2280.

26. Schock SN, Chandra NV, Sun Y, et al. Induction of necroptotic cell death by viral activation of the RIG-I or STING pathway. Cell Death Differ. 2017;24(4):615-625.

27. Ackermann M, Kim YO, Wagner WL, et al. Effects of nintedanib on the microvascular architecture in a lung fibrosis model. Angiogenesis. 2017;20(3):359-372.

28. Das N, Mandala A, Naaz S, et al. Melatonin protects against lipidinduced mitochondrial dysfunction in hepatocytes and inhibits stellate cell activation during hepatic fibrosis in mice. J Pineal Res. 2017;62(4): e12404.

29. Lee HJ, Jung YH, Choi GE, et al. BNIP3 induction by hypoxia stimulates FASN-dependent free fatty acid production enhancing therapeutic potential of umbilical cord blood-derived human mesenchymal stem cells. Redox Biol. 2017;13:426-443.

30. Alghanem AF, Wilkinson EL, Emmett MS, et al. RCAN1.4 regulates VEGFR-2 internalisation, cell polarity and migration in human microvascular endothelial cells. Angiogenesis. 2017;20(3):341-358.

31. Chang SH, Yeh YH, Lee JL, Hsu YJ, Kuo CT, Chen WJ. Transforming growth factor- $\beta$-mediated CD44/STAT3 signaling contributes to the development of atrial fibrosis and fibrillation. Basic Res Cardiol. 2017; 112(5):58.

32. Feng D, Wang B, Wang L, et al. Pre-ischemia melatonin treatment alleviated acute neuronal injury after ischemic stroke by inhibiting endoplasmic reticulum stress-dependent autophagy via PERK and IRE1 signalings. J Pineal Res. 2017;62(3):e12395.

33. Zhou H, Zhu P, Wang J, Zhu H, Ren J, Chen Y. Pathogenesis of cardiac ischemia reperfusion injury is associated with CK2 $\alpha$-disturbed mitochondrial homeostasis via suppression of FUNDC1-related mitophagy. Cell Death Differ. 2018;25(6):1080-1093.

34. Zhu P, Hu S, Jin Q, et al. Ripk3 promotes ER stress-induced necroptosis in cardiac IR injury: a mechanism involving calcium overload/XO/ROS/ mPTP pathway. Redox Biol. 2018;16:157-168.

35. Fuhrmann DC, Brüne B. Mitochondrial composition and function under the control of hypoxia. Redox Biol. 2017;12:208-215.

36. Zhang Y, Zhou H, Wu W, et al. Liraglutide protects cardiac microvascular endothelial cells against hypoxia/reoxygenation injury through the suppression of the SR-Ca(2+)-XO-ROS axis via activation of the GLP-1R/PI3K/Akt/survivin pathways. Free Radic Biol Med. 2016;95:278-292. 
37. Rossello X, Yellon DM. The RISK pathway and beyond. Basic Res Cardiol. 2017;113(1):2.

38. Zhang CS, Lin SC. AMPK promotes autophagy by facilitating mitochondrial fission. Cell Metab. 2016;23(3):399-401.

39. Kingery JR, Hamid T, Lewis RK, et al. Leukocyte iNOS is required for inflammation and pathological remodeling in ischemic heart failure. Basic Res Cardiol. 2017;112(2):19.

40. Ligeza J, Marona P, Gach N, et al. MCPIP1 contributes to clear cell renal cell carcinomas development. Angiogenesis. 2017;20(3):325-340.

41. Lee MS, Yin TC, Sung PH, Chiang JY, Sun CK, Yip HK. Melatonin enhances survival and preserves functional integrity of stem cells: a review. J Pineal Res. 2017;62(2):e12372.

42. Kang PT, Chen CL, Lin P, Chilian WM, Chen YR. Impairment of $\mathrm{pH}$ gradient and membrane potential mediates redox dysfunction in the mitochondria of the post-ischemic heart. Basic Res Cardiol. 2017;112(4):36.

43. Le Cras TD, Mobberley-Schuman PS, Broering M, Fei L, Trenor CC, Adams DM. Angiopoietins as serum biomarkers for lymphatic anomalies. Angiogenesis. 2017;20(1):163-173.

44. Zhou H, Yue Y, Wang J, Ma Q, Chen Y. Melatonin therapy for diabetic cardiomyopathy: a mechanism involving Syk-mitochondrial complex I-SERCA pathway. Cell Signal. 2018;47:88-100.

45. Hu Z, Cheng J, Xu J, Ruf W, Lockwood CJ. Tissue factor is an angiogenic-specific receptor for factor VII-targeted immunotherapy and photodynamic therapy. Angiogenesis. 2017;20(1):85-96.

46. Pan L, Zhou L, Yin W, Bai J, Liu R. miR-125a induces apoptosis, metabolism disorder and migration impairment in pancreatic cancer cells by targeting Mfn2-related mitochondrial fission. Int J Oncol. 2018; 53(1):124-136.
47. Zhu H, Jin Q, Li Y, et al. Melatonin protected cardiac microvascular endothelial cells against oxidative stress injury via suppression of IP3R$\left[\mathrm{Ca}^{2+}\right] \mathrm{c} / \mathrm{VDAC}-\left[\mathrm{Ca}^{2+}\right] \mathrm{m}$ axis by activation of MAPK/ERK signaling pathway. Cell Stress Chaperones. 2018;23(1):101-113.

48. Lei Q, Tan J, Yi S, Wu N, Wang Y, Wu H. Mitochonic acid 5 activates the MAPK-ERK-yap signaling pathways to protect mouse microglial BV-2 cells against TNF $\alpha$-induced apoptosis via increased Bnip3-related mitophagy. Cell Mol Biol Lett. 2018;23:14.

49. Shi C, Cai Y, Li Y, et al. Yap promotes hepatocellular carcinoma metastasis and mobilization via governing cofilin/F-actin/lamellipodium axis by regulation of JNK/Bnip3/SERCA/CaMKII pathways. Redox Biol. 2018;14:59-71.

50. Couto JA, Ayturk UM, Konczyk DJ, et al. A somatic GNA11 mutation is associated with extremity capillary malformation and overgrowth. Angiogenesis. 2017;20(3):303-306.

51. Zhou H, Wang J, Zhu P, Hu S, Ren J. Ripk3 regulates cardiac microvascular reperfusion injury: The role of IP3R-dependent calcium overload, XO-mediated oxidative stress and F-action/filopodia-based cellular migration. Cell Signal. 2018;45:12-22.

52. Wang X, Song Q. Mst1 regulates post-infarction cardiac injury through the JNK-Drp1-mitochondrial fission pathway. Cell Mol Biol Lett. 2018;23:21.
OncoTargets and Therapy

\section{Publish your work in this journal}

OncoTargets and Therapy is an international, peer-reviewed, open access journal focusing on the pathological basis of all cancers, potential targets for therapy and treatment protocols employed to improve the management of cancer patients. The journal also focuses on the impact of management programs and new therapeutic agents and protocols on

\section{Dovepress}

patient perspectives such as quality of life, adherence and satisfaction. The manuscript management system is completely online and includes a very quick and fair peer-review system, which is all easy to use. Visit http://www.dovepress.com/testimonials.php to read real quotes from published authors. 